\title{
Ru-Catalyzed Cyclization of Terminal Alkynals to Cycloalkenes
}

Jesús A. Varela, Carlos González-Rodríguez, Silvia G. Rubín, Luis Castedo, Carlos Saá*

Departamento de Química Orgánica y Unidad Asociada al CSIC, Facultad de Química, Universidad de Santiago de Compostela, 15782 Santiago de Compostela, Spain

\section{Supporting Information}

Index

I. General

II. Preparation of starting materials and catalysts

III. General procedure for the Ru-catalyzed reaction

IV. Optimization conditions for the Ru-catalyzed reactions.

V. Deuteration experiments

VI. Mechanistic Probes

VII. Spectral data

S5

VIII. Spectra 
I. General

Acetic acid (99.8\%, Fluka) was used as received. All other reagents and solvents: toluene, THF and $\mathrm{Et}_{2} \mathrm{O}(99.5-99.8 \%$, GC, over molecular sieves) were used as received without further purification, unless otherwise noted.

All reactions were carried out under an atmosphere of argon in flame-dried glassware with magnetic stirring, unless otherwise indicated.

\section{Preparation of starting materials and catalysts}

- Starting materials $\mathbf{1 a},{ }^{1} \mathbf{1 b},{ }^{2} \mathbf{1 c},{ }^{3} \mathbf{1} \mathbf{d}^{4}$ and $\mathbf{1 2},{ }^{5}$ were prepared following published procedures or with appropriate modifications.

- Alkynals 1e and 1f were synthesized following the procedure for $1 \mathbf{1 a},{ }^{1}$ but using 2-(2-bromoethyl)-1,3-dioxolane or 2-(3-chloropropyl)-1,3-dioxolane as first alkylating agents. For preparation of 9, 1-bromo-2-butyne were used as alkylating agent.

- Alkynes 7a, 7b and 4 were synthesized using methyl 2-chloroacetate, 2chloroacetonitrile and chloropropanone, respectively, as the first alkylating agents.

- $\left[\mathrm{Cp} * \mathrm{Ru}\left(\mathrm{CH}_{3} \mathrm{CN}\right)_{3}\right] \mathrm{PF}_{6},{ }^{6,7} \quad\left[\mathrm{CpRu}\left(\mathrm{CH}_{3} \mathrm{CN}\right)_{3}\right] \mathrm{PF}_{6},{ }^{7,8} \quad[\mathrm{CpRu}(\mathrm{dppm}) \mathrm{Cl}]{ }^{8}$ $\left[\mathrm{CpRu}\left(\mathrm{PPh}_{3}\right)_{2} \mathrm{Cl}\right],{ }^{9}$ and $[\mathrm{Cp} * \mathrm{Ru}(\mathrm{COD}) \mathrm{Cl}]^{10}$ were prepared following literature procedures.

- Vinyl acetate IIa was prepared by Pd-catalyzed alkylation ${ }^{11}$ of dimethyl 2-(2,2diethoxyethyl)malonate ${ }^{1}$ with the dimethyl acetal of acrolein: ${ }^{12}$. To a solution of dimethyl 2-(2,2-diethoxyethyl)malonate $(1.2 \mathrm{mmol})$, prop-2-ene-1,1-diyl diacetate $(1.2 \mathrm{mmol})$ and BSA $(1.3 \mathrm{mmol})$ in THF $(10 \mathrm{~mL})$ was added $\mathrm{Pd}\left(\mathrm{PPh}_{3}\right)_{4}$ $(0.12 \mathrm{mmol})$ and the resulting mixture was refluxed for $24 \mathrm{~h}$. Once the reaction mixture reached room temperature, water $(10 \mathrm{~mL})$ was added. The aqueous layer was extracted with diethyl ether $(3 \times 10 \mathrm{~mL})$, and the combined organic layers were dried over anhydrous $\mathrm{Na}_{2} \mathrm{SO}_{4}$ and concentrated under vacuum. The resulting residue was chromatographed on silica gel using ethyl ether/hexane (4:6) as eluent affording the final vinyl acetate IIa as a pale oil (37\%).

\footnotetext{
${ }^{1}$ Carretero, J. C.; Adrio, J. Synthesis. 2001, 12, 1888.

${ }^{2}$ Shintani, R.; Okamoto, K.; Otomaru, Y.; Ueyama, K.; Hayashi, T. J. Am. Chem. Soc. 2005, 127, 54.

${ }^{3}$ Feldman, K. S.; Bruendl, M. M.; Schildknegt, K.; Bohnstedt, A. C. J. Org. Chem. 1996, 61, 5440.

${ }^{4}$ Shizaki, M.; Hoshino, O. Tetrahedron 2000, 56, 8813.

${ }^{5}$ Bashiardes, G.; Safir, I.; Mohamed, A. Said; Barbot, F.; Laduranty, J. Org. Lett. 2003, 5, 4915.

${ }^{6}$ Trost, B. M.; Older, C. M. Organometallics 2002, $21,2544$.

7 Schenk, J. L.; McNair, A. M.; McCormick, F. B. Mann, K. R. Inorg. Chem. 1986, 25, 3501.

${ }^{8}$ Ashby, G. S.; Bruce, M. I. Tomkins, I. B.; Wallis, R. C. Aust. J. Chem. 1979, 32, 1003.

${ }^{9}$ Bruce, M. I.; Windsor, N. J. Aust. J. Chem. 1977, 30, 1601.

${ }^{10}$ Masuda, K.; Ohkita, H.; Kurumatani, S.; Itoh, K. Organometallics 1993, 12, 2221.

${ }^{11}$ Trost, B.M., Vercauteren J. Tetrahedron Lett. 1985, 26, 131.

${ }^{12}$ Deka, N., Kalita, D.J.. Borah, R., Sarma, J. C. J. Org. Chem. 1997, 62, 1563
} 


\section{General conditions for the Ru-catalyzed cyclization of alkynals}

Condition A. To a mixture of ruthenium catalyst $(5 \% \mathrm{mmol})$ in acetic acid $(4 \mathrm{~mL})$ was added alkynal 1a $(0.5 \mathrm{mmol})$ and then heated in an oil bath for $12-24 \mathrm{~h}$ at $90^{\circ} \mathrm{C}$. After reaching rt, diethyl ether was added to the solution and washed with water and saturated aqueous $\mathrm{NaHCO}_{3}$. The organic layer was dried over anhydrous $\mathrm{Na}_{2} \mathrm{SO}_{4}$ and concentrated under vacuum. The residue obtained was chromatographied on silica gel using ethyl acetate/hexane as eluents to afford the final cycloalkenes."

*Additives used for reaction of entry 6, (Table 1):

- Use of dppf (5\% mmol) gave exclusively $\mathbf{2 a}$ (see Table 1).

- Use of dppe, $\mathrm{P}(\mathrm{OPh})_{3}$ and $\mathrm{PPh}_{3}(5 \% \mathrm{mmol})$ gave no modification of 2a:3a ratio.

Condition B. To a mixture of ruthenium catalyst $(5 \%, \mathrm{mmol})$ in acetic acid $(4 \mathrm{~mL})$ in a screw-capped vial was added alkynal $1 \mathbf{1 a}(0.5 \mathrm{mmol})$ and then heated in an oil bath for 1$12 \mathrm{~h}$ at $130^{\circ} \mathrm{C}$. After reaching rt, diethyl ether was added to the solution and washed with water and saturated aqueous $\mathrm{NaHCO}_{3}$. The organic layer was dried over anhydrous $\mathrm{Na}_{2} \mathrm{SO}_{4}$ and concentrated under vacuum. The residue obtained was chromatographied on silica gel using ethyl acetate/hexane as eluents to afford the final products.

Condition C. ${ }^{13}$ To a mixture of $[\mathrm{CpRu}(\mathrm{dppm}) \mathrm{Cl}]\left(10 \%\right.$, mmol) in a mixture of $\mathrm{H}_{2} \mathrm{O}$ $(0.4 \mathrm{~mL})$ and 2-propanol $(1.3 \mathrm{~mL})$ in a screw-capped vial was added alkynal $1(0.5$ $\mathrm{mmol}$ ). The reaction mixture was stirred for $24 \mathrm{~h}$ at $130^{\circ} \mathrm{C}$. After reaching $\mathrm{rt}$, diethyl ether was added to the solution, dried over anhydrous $\mathrm{Na}_{2} \mathrm{SO}_{4}$ and concentrated under vacuum. The residue obtained was chromatographied on silica gel using ethyl acetate/hexane as eluents to afford the final aldehydes.

\footnotetext{
${ }^{13}$ Suzuki, T.; Tokunaga, M.; Wakatsuki, Y. Org. Lett. 2001, 3, 735
} 


\section{Optimization conditions for the Ru-catalyzed reactions.}

Besides the experimental conditions showed in the main text, the following catalyst and solvents were also used for the optimization of the Ru-catalyzed cyclization of alkynal 1a (Table 1).

Table 1. Ru-catalyzed cyclization of alkynal $1 \mathbf{a}\left[\mathrm{X}=\mathrm{C}\left(\mathrm{CO}_{2} \mathrm{Me}\right)_{2}, \mathrm{n}=1\right]$.

\begin{tabular}{ccccccc}
\hline entry & catalyst $^{\mathrm{a}}$ & solvent & $\mathrm{t}^{\circ} \mathrm{C}$ & time & yield $^{\mathrm{b}}$ & $\mathbf{2 a}: \mathbf{3 a}$ \\
\hline 1 & $\mathrm{Cp} * \mathrm{RuCl}(\mathrm{cod})$ & $\mathrm{AcOH}$ & 90 & $5.5 \mathrm{~h}$ & $94 \%^{\mathrm{c}}$ & $80: 20$ \\
2 & $\mathrm{CpRuCl}\left(\mathrm{PPh}_{3}\right)_{2}$ & $\mathrm{AcOH}$ & 90 & $24 \mathrm{~h}$ & $58 \%$ & $95: 5$ \\
3 & $\mathrm{RuCl}_{2}\left(\mathrm{PPh}_{3}\right)_{3}$ & $\mathrm{AcOH}$ & 90 & $24 \mathrm{~h}$ & $20 \%$ & $95: 5$ \\
4 & $\left(\mathrm{ArRuCl}_{2}\right)_{2}$ & $\mathrm{AcOH}$ & 90 & $24 \mathrm{~h}$ & $50 \%$ & $95: 5$ \\
5 & {$\left[\mathrm{CpRuL}_{3}\right] \mathrm{PF}_{6}$} & $\mathrm{AcOH} / \mathrm{H}_{2} \mathrm{O} 90: 10$ & 90 & $24 \mathrm{~h}$ & $90 \%$ & $96: 4$ \\
6 & {$\left[\mathrm{CpRuL}_{3}\right] \mathrm{PF}_{6}$} & $\mathrm{AcOH} / \mathrm{H}_{2} \mathrm{O} \mathrm{50:50}$ & 90 & $24 \mathrm{~h}$ & $55 \%$ & $94: 6$ \\
7 & {$\left[\mathrm{CpRuL}_{3}\right] \mathrm{PF}_{6}$} & $\mathrm{C}_{2} \mathrm{H}_{5} \mathrm{COOH}$ & 90 & $24 \mathrm{~h}$ & $90 \%$ & $90: 10$ \\
\hline
\end{tabular}

${ }^{\mathrm{a}} \mathrm{L}=\mathrm{CH}_{3} \mathrm{CN}, \mathrm{Ar}=p$-cymene. ${ }^{\mathrm{b}}$ Isolated yields. ${ }^{\mathrm{c}} \mathrm{GC}$ yields.

\section{Deuteration experiments.}

The reaction was carried out in an NMR tube equipped with a $J$ Young valve under argon atmosphere. Benzaldehyde $12(40 \mathrm{mg}, 0.25 \mathrm{mmol})$ was added over a mixture of $\mathrm{Cp} * \mathrm{Ru}\left(\mathrm{CH}_{3} \mathrm{CN}\right)_{3} \mathrm{PF}_{6}(6 \mathrm{mg}, 0.013 \mathrm{mmol})$ in $\mathrm{CD}_{3} \mathrm{COOD}(0.75 \mathrm{~mL})$, and the mixture was heated at $90^{\circ} \mathrm{C}$. The course of the experiment was monitorized by ${ }^{1} \mathrm{H}$ NMR $(300 \mathrm{MHz})$ during 12h. After partial deuteration of starting alkynal 12 (S27), ${ }^{14}$ evolution to the deuterated chromene $\mathbf{1 3}$ was observed.

\section{Mechanistic probes.}

- A solution of vinyl acetate IIa $(0.5 \mathrm{mmol})$ in $\mathrm{AcOH}(4 \mathrm{~mL})$ was heated at $90^{\circ} \mathrm{C}$ in an oil bath. The reaction was monitored by GC-MS, showing a slow (24 h) quantitative conversion to the cyclic $\alpha, \beta$-unsaturated aldehyde 11a.

- A solution of vinyl acetate IIa $(0.5 \mathrm{mmol})$ and $\mathrm{CpRu}\left(\mathrm{CH}_{3} \mathrm{CN}\right)_{3} \mathrm{PF}_{6}(0.05 \mathrm{mmol})$ in $\mathrm{AcOH}(4 \mathrm{~mL})$ was heated at $90^{\circ} \mathrm{C}$ in an oil bath. The reaction was monitored by GC-MS showing a faster conversion (4-5 h) to the cyclic $\alpha, \beta$-unsaturated aldehyde $11 a$.

- To a mixture of $\mathrm{CpRu}\left(\mathrm{CH}_{3} \mathrm{CN}\right)_{3} \mathrm{PF}_{6}(0.05 \mathrm{mmol})$ in $\mathrm{AcOH}(4 \mathrm{~mL})$ placed in a screw-capped vial was added the $\alpha, \beta$-unsaturated aldehyde 11a $(0.5 \mathrm{mmol})$. The mixture was heated at $90^{\circ} \mathrm{C}$ in an oil bath and monitored by GC-MS. After $24 \mathrm{~h}$ the starting aldehyde 11a was recovered unaffected.

A second run of the same experiment performed at $130{ }^{\circ} \mathrm{C}$ gave the same result.

\footnotetext{
${ }^{14}$ Tokunaga, M.; Suzuki, T.; Koga, N.; Fukushima, T.; Horiuchi, A.; Wakatsuki, Y. J. Am. Chem. Soc. 2001, 123, 11917.
} 
VII. Spectral data.

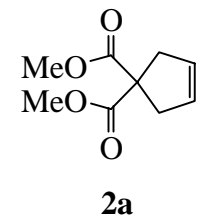

Dimethyl cyclopent-3-ene-1, 1-dicarboxylate (2a) ${ }^{15}$

${ }^{1} \mathrm{H}$ NMR $\left(300 \mathrm{MHz}, \mathrm{CDCl}_{3}\right), \delta(\mathrm{ppm}): 5.61(\mathrm{~s}, 1 \mathrm{H}), 3.74(\mathrm{~s}, 6 \mathrm{H}), 3.02(\mathrm{~s}, 4 \mathrm{H}),{ }^{13} \mathrm{C}$ NMR, DEPT (75 MHz, CDCl 3$), \delta(\mathrm{ppm}): 172.6(2 \times \mathrm{CO}), 127.8(2 \times \mathrm{CH}), 58.7(\mathrm{C}), 52.8$ $\left(2 \times \mathrm{CH}_{3}\right), 40.9\left(2 \times \mathrm{CH}_{2}\right) . \mathrm{MS}, \mathrm{m} / \mathrm{z}$ (\% relative intensity): $184\left(\mathrm{M}^{+}, 10\right), 152(21), 124$ (100), 93 (61). HRMS calculated for $\mathrm{C}_{9} \mathrm{H}_{12} \mathrm{O}_{4}$ : 184.0735; found: 184.0742 .

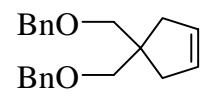

$2 \mathbf{b}$

4, 4-Bis (benzyloxymethyl)cyclopent-1-ene $(2 \mathrm{~b})^{16}$

${ }^{1} \mathrm{H}$ NMR (300 MHz, $\left.\mathrm{CDCl}_{3}\right), \delta(\mathrm{ppm}): 7.32-7.28(\mathrm{~m}, 10 \mathrm{H}), 5.58(\mathrm{~s}, 2 \mathrm{H}), 4.53(\mathrm{~s}, 4 \mathrm{H})$, $3.44(\mathrm{~s}, 4 \mathrm{H}), 2.24(\mathrm{~s}, 4 \mathrm{H}) .{ }^{13} \mathrm{C}$ NMR, DEPT $\left(75 \mathrm{MHz}, \mathrm{CDCl}_{3}\right), \delta(\mathrm{ppm}): 138.9(\mathrm{C})$, $128.9(\mathrm{CH}), 128.2(2 \times \mathrm{CH}), 127.4(2 \times \mathrm{CH}), 127.3(\mathrm{CH}), 74.1\left(2 \times \mathrm{CH}_{2}\right), 73.2\left(2 \times \mathrm{CH}_{2}\right)$, $46.7(\mathrm{C}), 39.4\left(2 \times \mathrm{CH}_{2}\right)$. MS, m/z (\% relative intensity): $309\left(\mathrm{M}^{+}+1,77\right), 308(9), 307$ (24), 183 (32), 182 (20), 181 (100). HRMS calculated for $\mathrm{C}_{21} \mathrm{H}_{25} \mathrm{O}_{2}$ : 309.1854; found: 309.1864 .

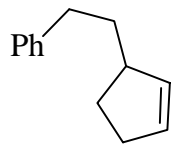

2c

1-(2-(Cyclopent-2-enyl)ethyl)benzene (2c) ${ }^{17}$

${ }^{1} \mathrm{H}$ NMR $\left(300 \mathrm{MHz}, \mathrm{CDCl}_{3}\right), \delta(\mathrm{ppm}): 7.31-7.14(\mathrm{~m}, 5 \mathrm{H}), 5.77-5.67(\mathrm{~m}, 2 \mathrm{H}), 2.65(\mathrm{t}, J=$ $7.8 \mathrm{~Hz}, 2 \mathrm{H}), 2.38-2.27(\mathrm{~m}, 2 \mathrm{H}), 2.14-1.99(\mathrm{~m}, 1 \mathrm{H}), 1.92-1.41(\mathrm{~m}, 4 \mathrm{H}) .{ }^{13} \mathrm{C}$ NMR, DEPT $\left(75 \mathrm{MHz}, \mathrm{CDCl}_{3}\right), \delta(\mathrm{ppm}): 142.8(\mathrm{C}), 134.9(\mathrm{CH}), 130.5(\mathrm{CH}), 128.4(2 \times C H), 128.3$ (2xCH), $125.6(\mathrm{CH}), 45.2(\mathrm{CH}), 37.9\left(\mathrm{CH}_{2}\right), 34.3\left(\mathrm{CH}_{2}\right), 31.9\left(\mathrm{CH}_{2}\right), 29.8\left(\mathrm{CH}_{2}\right) . \mathrm{MS}$, $\mathrm{m} / \mathrm{z}$ (\% relative intensity): $173\left(\mathrm{M}^{+}+1,21\right), 172$ (6), 171 (21), 131 (13), 119 (33), 105 (15), 91 (100). HRMS calculated for $\mathrm{C}_{13} \mathrm{H}_{17}$ : 173.1330; found: 173.1329.

\footnotetext{
${ }^{15}$ Depres, J. P.; Greene, A. E. J. Org. Chem. 1984, 49, 928.

${ }^{16}$ Legraverend, M.; Huel, C.; Bisagni, E. J. Chem. Res., Synopses. 1990, 4, 102.

${ }^{17}$ Ellis-Davies, G. C. R.; Gilbert, A.; Heath, P.; Lane, J. C.; Warrington, J. V.; Westover, D. L. J. Chem Soc, Perkin Trans. 2 1984, 11, 1833.
} 


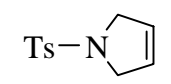

2d

2,5- Dihydro-1-tosyl-1H-pyrrole (2d) ${ }^{18}$

${ }^{1} \mathrm{H}$ NMR (250 MHz, $\left.\mathrm{CDCl}_{3}\right), \delta(\mathrm{ppm}): 7.72(\mathrm{~d}, J=8.3 \mathrm{~Hz}, 2 \mathrm{H}), 7.32(\mathrm{~d}, J=7.8 \mathrm{~Hz}, 2 \mathrm{H})$, $5.65(\mathrm{~s}, 2 \mathrm{H}), 4.12(\mathrm{~s}, 4 \mathrm{H}), 2.43(\mathrm{~s}, 3 \mathrm{H}) .{ }^{13} \mathrm{C} \mathrm{NMR}, \mathrm{DEPT}\left(63 \mathrm{MHz}, \mathrm{CDCl}_{3}\right), \delta(\mathrm{ppm})$ : $143.4(\mathrm{C}), 134.3(\mathrm{C}), 129.7(2 \times \mathrm{CH}), 127.4(2 \times \mathrm{CH}), 125.4(2 \times \mathrm{CH}), 54.8\left(2 \times \mathrm{CH}_{2}\right), 21.5$ $\left(\mathrm{CH}_{3}\right) . \mathrm{MS}, \mathrm{m} / \mathrm{z}$ (\% relative intensity): $224\left(\mathrm{M}^{+}+1,100\right), 223$ (6), 222 (17), 149 (23). HRMS calculated for $\mathrm{C}_{11} \mathrm{H}_{14} \mathrm{~N}_{1} \mathrm{O}_{2} \mathrm{~S}: 224.0745$; found: 224.0748 .

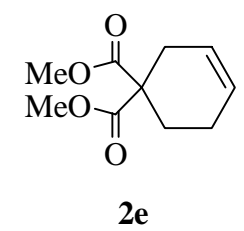

Dimethyl cyclohex-3-ene-1,1-dicarboxylate $(2 \mathrm{e})^{19}$

${ }^{1} \mathrm{H}$ NMR (250 MHz, $\left.\mathrm{CDCl}_{3}\right), \delta(\mathrm{ppm}): 5.65$ (s, 2H), 3.71 (s, 6H), 2.56 (s, 2H), 2.17-2.06 $(\mathrm{m}, 4 \mathrm{H}) .{ }^{13} \mathrm{C}$ NMR, DEPT $\left(63 \mathrm{MHz}, \mathrm{CDCl}_{3}\right), \delta(\mathrm{ppm}): 171.9(2 \times \mathrm{CO}), 126.0(\mathrm{CH})$, $123.8(\mathrm{CH}), 53.0(\mathrm{C}), 52.6\left(2 \times \mathrm{CH}_{3}\right), 30.6\left(\mathrm{CH}_{2}\right), 27.6\left(\mathrm{CH}_{2}\right), 22.4\left(\mathrm{CH}_{2}\right) . \mathrm{MS}, \mathrm{m} / \mathrm{z}(\%$ relative intensity): $199\left(\mathrm{M}^{+}+1,28\right), 197$ (7), 167 (95), 139 (100), 79 (15). HRMS calculated for $\mathrm{C}_{10} \mathrm{H}_{15} \mathrm{O}_{4}$ : 199.0970 ; found: 199.0978 .

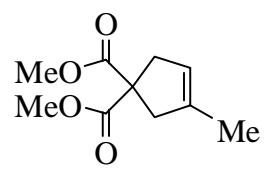

5

Dimethyl 3-methylcyclopent-3-ene-1,1-dicarboxylate $(\mathbf{5})^{20}$

${ }^{1} \mathrm{H}$ NMR (400 MHz, $\mathrm{CDCl}_{3}$ ), $\delta(\mathrm{ppm}): 5.18$ (m, 1H), 3.73 (s, 6H), 2.97 (m, 2H), 2.91 (broad s, 2H), 1.70 (broad s, 3H). $\left.{ }^{13} \mathrm{C} \mathrm{NMR,} \mathrm{DEPT} \mathrm{(100} \mathrm{MHz,} \mathrm{CDCl}_{3}\right), \delta(\mathrm{ppm}): 172.8$ $(2 \times \mathrm{CO}), 137.4(\mathrm{C}), 121.2(\mathrm{CH}), 59.3(\mathrm{C}), 52.7\left(2 \times \mathrm{CH}_{3}\right), 44.7\left(\mathrm{CH}_{2}\right), 40.9\left(\mathrm{CH}_{2}\right), 16.0$ $\left(\mathrm{CH}_{3}\right) . \mathrm{MS}, \mathrm{m} / \mathrm{z}$ (\% relative intensity): $199\left(\mathrm{M}^{+}+1,17\right), 198(2), 197$ (6), $167(37), 139$ (100), $123(8)$.

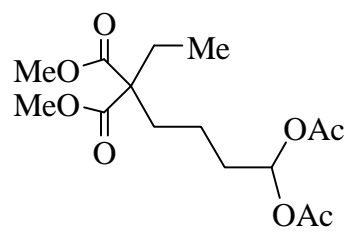

6

Dimethyl-2-[4,4-di(methylcarbonyloxy)butyl]-2-ethylmalonate (6)

\footnotetext{
${ }^{18}$ Kimura, M.; Harayama, H.; Tanaka, S.; Tamaru, Y. J.Chem. Soc., Chem. Commun. 1994, 21, 2531.

${ }^{19}$ Baylouny, R. A. J. Am. Chem. Soc. 1971, 93, 4621.

${ }^{20}$ Furstner, A.; Ackermann, L. Chem. Commun. 1999, 1, 95.
} 
${ }^{1} \mathrm{H}$ NMR (400 MHz, $\left.\mathrm{CDCl}_{3}\right), \delta(\mathrm{ppm}): 6.75(\mathrm{t}, J=5.6 \mathrm{~Hz}, 1 \mathrm{H}), 3.72(\mathrm{~s}, 6 \mathrm{H}), 2.07(\mathrm{~s}$, $6 \mathrm{H}), 1.98-186(\mathrm{~m}, 4 \mathrm{H}), 1.78(\mathrm{~m}, 2 \mathrm{H}), 1.31-1.18(\mathrm{~m}, 2 \mathrm{H}), 0.81(\mathrm{t}, J=7.5 \mathrm{~Hz}, 3 \mathrm{H}){ }^{13} \mathrm{C}$ NMR, DEPT (100 MHz, $\left.\mathrm{CDCl}_{3}\right), \delta(\mathrm{ppm}): 171.9$ (2xCO), 168.9 (2xCO), $90.0(\mathrm{CH})$, $57.9(\mathrm{C}), 52.3\left(2 \mathrm{xCH}_{3}\right), 33.2\left(\mathrm{CH}_{2}\right), 31.6\left(\mathrm{CH}_{2}\right), 25.6\left(\mathrm{CH}_{2}\right), 20.8\left(2 \times \mathrm{CH}_{3}\right), 18.1\left(\mathrm{CH}_{2}\right)$, $8.5\left(\mathrm{CH}_{3}\right), \mathrm{MS}, \mathrm{m} / \mathrm{z}$ (\% relative intensity): $355\left(\mathrm{M}^{+}+\mathrm{Na}, 6\right), 295$ (14), $254(20), 253$ (100), 179 (13). HRMS calculated for $\mathrm{C}_{15} \mathrm{H}_{24} \mathrm{O}_{8} \mathrm{Na}$ : 355.1369; found: 355.1363 .

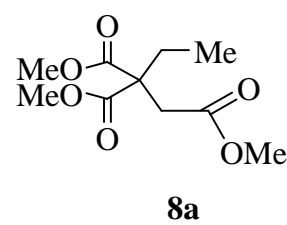

Trimethyl butane-1,2,2-tricarboxylate (8a)

${ }^{1} \mathrm{H}$ NMR (250 MHz, CDCl $), \delta(\mathrm{ppm}): 3.72$ (s, 6H), 3.64 (s, 3H), 2.94 (s, 2H), 2.01 (q, $J=7.5 \mathrm{~Hz}, 2 \mathrm{H}), 0.84(\mathrm{t}, J=7.5 \mathrm{~Hz}, 3 \mathrm{H}) .{ }^{13} \mathrm{C} \mathrm{NMR}$, DEPT $\left(63 \mathrm{MHz}, \mathrm{CDCl}_{3}\right), \delta(\mathrm{ppm})$ : $170.9(2 \times \mathrm{CO}), 170.8(\mathrm{CO}), 56.0(\mathrm{C}), 52.7\left(2 \times \mathrm{CH}_{3}\right), 51.8\left(\mathrm{CH}_{3}\right), 36.9\left(\mathrm{CH}_{2}\right), 26.5\left(\mathrm{CH}_{2}\right)$, $8.8\left(\mathrm{CH}_{3}\right) . \mathrm{MS}, \mathrm{m} / \mathrm{z}$ (\% relative intensity): $233\left(\mathrm{M}^{+}+1,6\right), 202$ (10), 201 (100), 141 (3). HRMS calculated for $\mathrm{C}_{10} \mathrm{H}_{17} \mathrm{O}_{6} 233.1025$; found: 233.1025 .

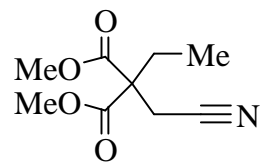

$\mathbf{8 b}$

\section{Dimethyl 2-(cyanomethyl)-2- ethylmalonate (8b)}

${ }^{1} \mathrm{H}$ NMR (250 MHz, $\left.\mathrm{CDCl}_{3}\right), \delta(\mathrm{ppm}): 3.72(\mathrm{~s}, 6 \mathrm{H}), 2.90(\mathrm{~s}, 2 \mathrm{H}), 2.09$ (q, J= $7.5 \mathrm{~Hz}$, $2 \mathrm{H}), 0.85(\mathrm{t}, J=7.5 \mathrm{~Hz}, 3 \mathrm{H}) \cdot{ }^{13} \mathrm{C}$ NMR, DEPT $\left(75 \mathrm{MHz}, \mathrm{CDCl}_{3}\right), \delta(\mathrm{ppm}): 169.2$ $(2 \times \mathrm{CO}), 116.2(\mathrm{CN}), 55.8(\mathrm{C}), 53.3\left(2 \times \mathrm{CH}_{3}\right), 26.1\left(\mathrm{CH}_{2}\right), 21.3\left(\mathrm{CH}_{2}\right), 8.5\left(\mathrm{CH}_{3}\right) . \mathrm{MS}$, $\mathrm{m} / \mathrm{z}$ (\% relative intensity): $200\left(\mathrm{M}^{+}+1,100\right), 169(4), 168(40), 140$ (13).

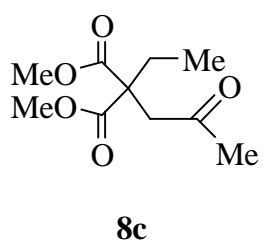

\section{Dimethyl 2-ethyl-2-(2-oxopropyl)malonate $(8 c)^{21}$}

${ }^{1} \mathrm{H}$ NMR (400 MHz, $\mathrm{CDCl}_{3}$ ), $\delta(\mathrm{ppm}): 3.72(\mathrm{~s}, 6 \mathrm{H}), 3.10(\mathrm{~s}, 2 \mathrm{H}), 2.16$ (s, 3H), 2.04 (q, $J=7.6 \mathrm{~Hz}, 2 \mathrm{H}), 0.83(\mathrm{t}, J=7.6 \mathrm{~Hz}, 3 \mathrm{H}) .{ }^{13} \mathrm{C} \mathrm{NMR}$, DEPT $\left(100 \mathrm{MHz}, \mathrm{CDCl}_{3}\right), \delta(\mathrm{ppm})$ : $205.2(\mathrm{CO}), 171.3(2 \times \mathrm{CO}), 55.7(\mathrm{C}), 52.6\left(2 \times \mathrm{CH}_{3}\right), 45.6\left(\mathrm{CH}_{2}\right), 30.3\left(\mathrm{CH}_{3}\right), 26.7\left(\mathrm{CH}_{2}\right)$, $9.1\left(\mathrm{CH}_{3}\right) . \mathrm{MS}, \mathrm{m} / \mathrm{z}$ (\% relative intensity): $217\left(\mathrm{M}^{+}+1,6\right), 186(10), 185$ (100), 157 (7), 129 (17). HRMS calculated for $\mathrm{C}_{10} \mathrm{H}_{17} \mathrm{O}_{5}$ : 217.1076; found: 217.1080 .

\footnotetext{
${ }^{21}$ Hosomi, A.; Shirahata, A.; Araki, Y.; Sakurai, H. J. Org. Chem. 1981, 46, 4631.
} 


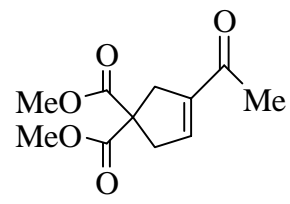

10

Dimethyl 3-acetylcyclopent-3-ene-1,1-dicarboxylate $(10)^{22}$

${ }^{1} \mathrm{H}$ NMR $\left(300 \mathrm{MHz}, \mathrm{CDCl}_{3}\right), \delta(\mathrm{ppm}): 6.52(\mathrm{~m}, 1 \mathrm{H}), 3.68(\mathrm{~s}, 6 \mathrm{H}), 3.20-3.19(\mathrm{~m}, 2 \mathrm{H})$, 3.18-3.16 (m, 2H), $2.25(\mathrm{~s}, 3 \mathrm{H}) .{ }^{13} \mathrm{C}$ NMR, DEPT (75 MHz, $\left.\mathrm{CDCl}_{3}\right), \delta(\mathrm{ppm}): 195.6$ (C), $171.7(2 \times \mathrm{CO}), 142.6(\mathrm{C}), 139.8(\mathrm{CH}), 58.1(\mathrm{C}), 52.9\left(2 \times \mathrm{CH}_{3}\right), 41.5\left(\mathrm{CH}_{2}\right), 38.8$ $\left(\mathrm{CH}_{2}\right), 26.3\left(\mathrm{CH}_{3}\right) . \mathrm{MS}, \mathrm{m} / \mathrm{z}$ (\% relative intensity): $227\left(\mathrm{M}^{+}+1,89\right), 226(6), 195(100)$, 167 (69), 135 (26), 125 (77). HRMS calculated for $\mathrm{C}_{11} \mathrm{H}_{15} \mathrm{O}_{5}$ : 227.0919; found: 227.0913 .

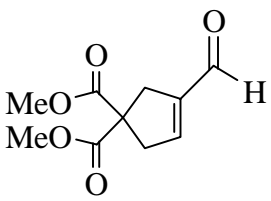

11a

Dimethyl 3-formylcyclopent-3-ene-1,1-dicarboxylate (11a)

${ }^{1} \mathrm{H}$ NMR (300 MHz, CDCl $), \delta(\mathrm{ppm}): 9.72(\mathrm{~s}, 1 \mathrm{H}), 6.71(\mathrm{~m}, 1 \mathrm{H}), 3.76(\mathrm{~s}, 6 \mathrm{H}), 3.31(\mathrm{~m}$, 2H), $3.23(\mathrm{~m}, 2 \mathrm{H}) .{ }^{13} \mathrm{C}$ NMR, DEPT $\left(75 \mathrm{MHz}, \mathrm{CDCl}_{3}\right), \delta(\mathrm{ppm}): 188.5(\mathrm{CHO}), 171.5$ $(2 \times \mathrm{CO}), 147.5(\mathrm{CH}), 144.2(\mathrm{C}), 58.3(\mathrm{C}), 53.1\left(2 \times \mathrm{CH}_{3}\right), 41.5\left(\mathrm{CH}_{2}\right), 37.0\left(\mathrm{CH}_{2}\right) . \mathrm{MS}$, $\mathrm{m} / \mathrm{z}$ (\% relative intensity): $213\left(\mathrm{M}^{+}+1,84\right), 182$ (10), 181 (100), 153 (98). HRMS calculated for $\mathrm{C}_{10} \mathrm{H}_{13} \mathrm{O}_{5}$ : 213.0762; found: 213.0762 .<smiles>COC(=O)C1(C(=O)OC)C=CC=CC1</smiles>

11e

Dimethyl 4-formylcyclohex-3-ene-1,1-dicarboxylate (11e)

${ }^{1} \mathrm{H}$ NMR (250 MHz, $\mathrm{CDCl}_{3}$ ), $\delta$ (ppm): 9.45 (s, 1H), 6.77 (m, 1H), 3.73 (s, 6H), 2.78 (m, 2H), 2.49-2.41 (m, 2H), $2.21(\mathrm{t}, J=6.4 \mathrm{~Hz}, 2 \mathrm{H}) .{ }^{13} \mathrm{C}$ NMR, DEPT $\left(63 \mathrm{MHz}, \mathrm{CDCl}_{3}\right), \delta$ (ppm): $192.8(\mathrm{CHO}), 171.2(2 \times \mathrm{CO}), 148.6(\mathrm{CH}), 138.6(\mathrm{C}), 52.7\left(2 \times \mathrm{CH}_{3}\right), 52.2(\mathrm{C})$, $27.2\left(\mathrm{CH}_{2}\right), 27.1\left(\mathrm{CH}_{2}\right), 23.7\left(\mathrm{CH}_{2}\right) . \mathrm{MS}, \mathrm{m} / \mathrm{z}$ (\% relative intensity): $227\left(\mathrm{M}^{+}+1,52\right)$, 226 (6), 209 (32), 196 (11), 195 (100), 167 (60).

${ }^{22}$ Beifuss, U.; Ledderhose, S. Synlett. 1995, 9, 938. 


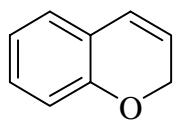

$\mathbf{2 H}$-chromene $(\mathbf{1 3})^{23}$

13

${ }^{1} \mathrm{H}$ NMR $\left(300 \mathrm{MHz}, \mathrm{CDCl}_{3}\right), \delta(\mathrm{ppm}): 7.09(\mathrm{td}, J=7.5,1.7 \mathrm{~Hz}, 1 \mathrm{H}), 6.95(\mathrm{dd}, J=7.5,1.7$ $\mathrm{Hz}, 1 \mathrm{H}), 6.85(\mathrm{td}, J=7.5,1.2 \mathrm{~Hz}, 1 \mathrm{H}), 6.76(\mathrm{~d}, J=7.4 \mathrm{~Hz}, 1 \mathrm{H}), 6.41(\mathrm{td}, J=9.8,1.7 \mathrm{~Hz}$, $1 \mathrm{H}), 5.76(\mathrm{td}, J=9.8,3.6 \mathrm{~Hz}, 1 \mathrm{H}), 4.81(\mathrm{dd}, J=3.6,1.7 \mathrm{~Hz}, 1 \mathrm{H}) .{ }^{13} \mathrm{C}$ NMR, DEPT $(75$ $\left.\mathrm{MHz}, \mathrm{CDCl}_{3}\right), \delta(\mathrm{ppm}): 154.0(\mathrm{C}), 129.1(\mathrm{CH}), 126.5(\mathrm{CH}), 124.5(\mathrm{CH}), 122.4(\mathrm{C})$, $121.9(\mathrm{CH}), 121.3(\mathrm{CH}), 115.7(\mathrm{CH}), 65.5\left(\mathrm{CH}_{2}\right) . \mathrm{MS}, \mathrm{m} / \mathrm{z}$ (\% relative intensity): 132 $\left(\mathrm{M}^{+}, 27\right), 131$ (100), 78 (58), 63 (94). HRMS calculated for $\mathrm{C}_{9} \mathrm{H}_{8} \mathrm{O}: 132.0575$; found: 132.0574 .

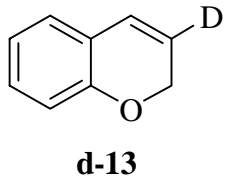

\section{3-Deutero-2H-chromene $(\mathrm{d}-13)^{24}$}

${ }^{1} \mathrm{H}$ NMR (300 MHz, $\mathrm{CDCl}_{3}$ ), $\delta(\mathrm{ppm}): 7.08$ (td, $\left.J=7.5,1.7 \mathrm{~Hz}, 1 \mathrm{H}\right), 6.94$ (dd, $J=7.5,1.7$ $\mathrm{Hz}, 1 \mathrm{H}), 6.84(\mathrm{td}, J=7.5,1.2 \mathrm{~Hz}, 1 \mathrm{H}), 6.75(\mathrm{~d}, J=7.5 \mathrm{~Hz}, 1 \mathrm{H}), 6.40(\mathrm{~s}, 1 \mathrm{H}), 4.80$ (d, $J=$ $1.7 \mathrm{~Hz}, 1 \mathrm{H}) .{ }^{13} \mathrm{C}$ NMR, DEPT $\left(75 \mathrm{MHz}, \mathrm{CDCl}_{3}\right), \delta(\mathrm{ppm}): 154.0(\mathrm{C}), 129.1(\mathrm{CH}), 126.5$ $(\mathrm{CH}), 124.4(\mathrm{CH}), 122.4(\mathrm{C}), 121.9(\mathrm{C}), 121.3(\mathrm{CH}), 115.7(\mathrm{CH}), 65.5\left(\mathrm{CH}_{2}\right) . \mathrm{MS}, \mathrm{m} / \mathrm{z}$ (\% relative intensity): $134\left(\mathrm{M}^{+}+1,100\right), 133(29), 132$ (8), 131 (3), 106 (6).

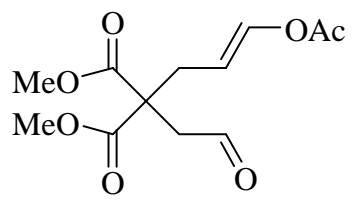

Ila

(E)-dimethyl 2-(3-acetoxyallyl)-2-(2-oxoethyl)malonate (IIa)

${ }^{1} \mathrm{H}$ NMR $\left(250 \mathrm{MHz}, \mathrm{CDCl}_{3}\right.$ ), $\delta(\mathrm{ppm}): 9.69(\mathrm{~s}, 1 \mathrm{H}), 7.06(\mathrm{dt}, J=12.4,1.2 \mathrm{~Hz}, 1 \mathrm{H}), 5.26$ $(\mathrm{dt}, J=12.4,8.2 \mathrm{~Hz}, 1 \mathrm{H}), 3.74(\mathrm{~s}, 6 \mathrm{H}), 2.98(\mathrm{~s}, 2 \mathrm{H}), 2.70(\mathrm{dd}, J=8.2,1.2 \mathrm{~Hz}, 2 \mathrm{H}), 2.09$ (s, 3H). ${ }^{13} \mathrm{C}$ NMR, DEPT (63 MHz, $\left.\mathrm{CDCl}_{3}\right), \delta$ (ppm): 198.4 (CHO), $170.1(2 \times \mathrm{C}), 167.7$ (C), $138.6(\mathrm{CH}), 108.2(\mathrm{CH}), 54.9(\mathrm{C}), 52.9\left(2 \times \mathrm{CH}_{3}\right), 46.1\left(\mathrm{CH}_{2}\right), 32.1\left(\mathrm{CH}_{2}\right), 20.5$ $\left(\mathrm{CH}_{3}\right) . \mathrm{MS}, \mathrm{m} / \mathrm{z}$ (\% relative intensity): $273\left(\mathrm{M}^{+}+1,1\right), 213$ (100), 199 (38), 181 (54), 153 (87).

\footnotetext{
23 a) Baranton, F.; Fontaine, G.; Maitte, P. Bull. Soc. Chim. Fr. 1968, 10, 4203. b) Koch-Pomeranz, U.; Hansen, H. J.; Schmid, H. Helv. Chim. Acta. 1973, 56, 2981.

${ }^{24}$ Spilker, R.; Gruetzmacher, H. F. Org. Mass Spectrom. 1989, 24, 47.
} 
VIII. Spectra.

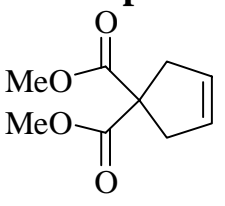

$2 a$

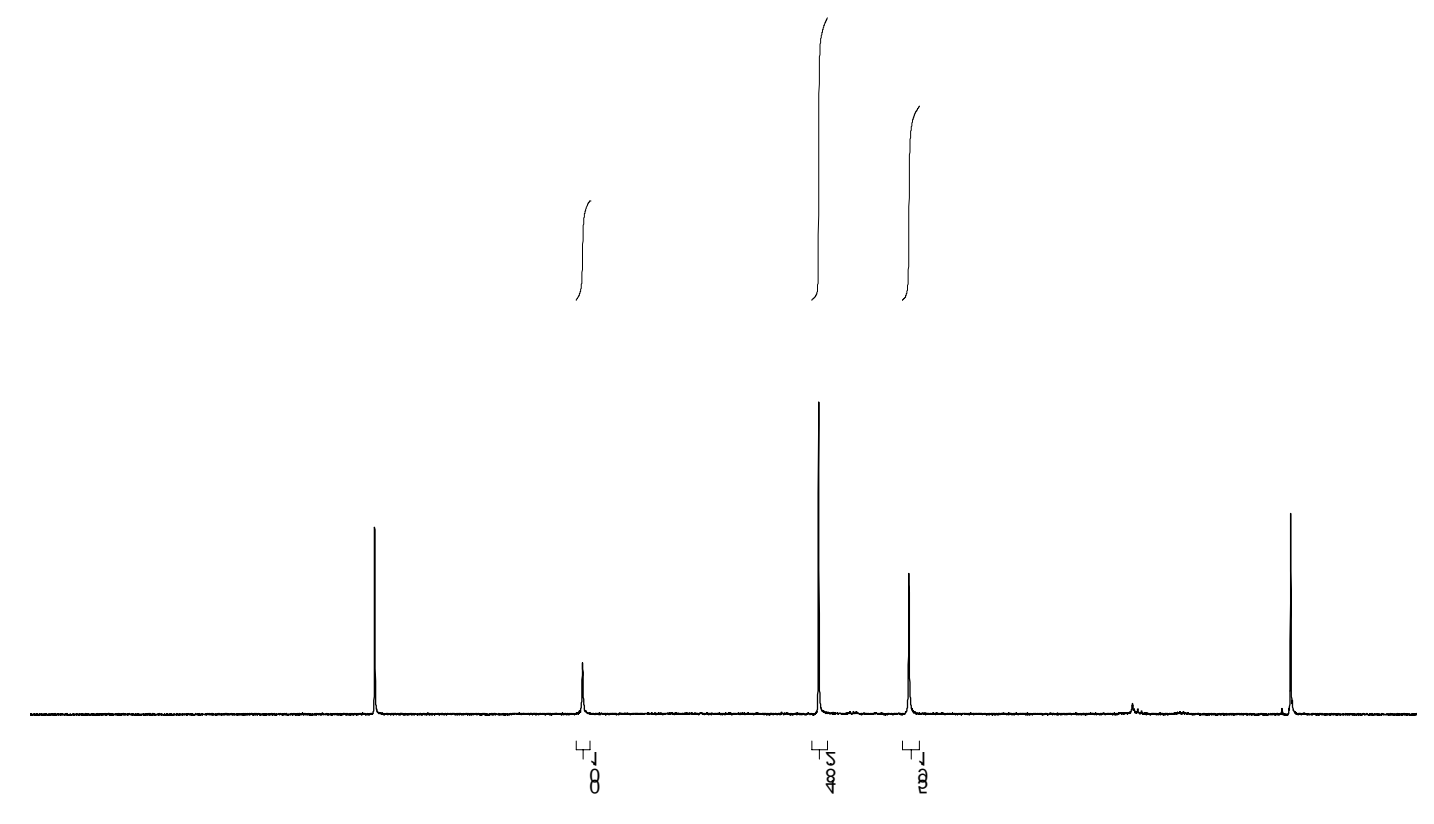

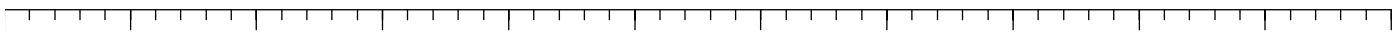
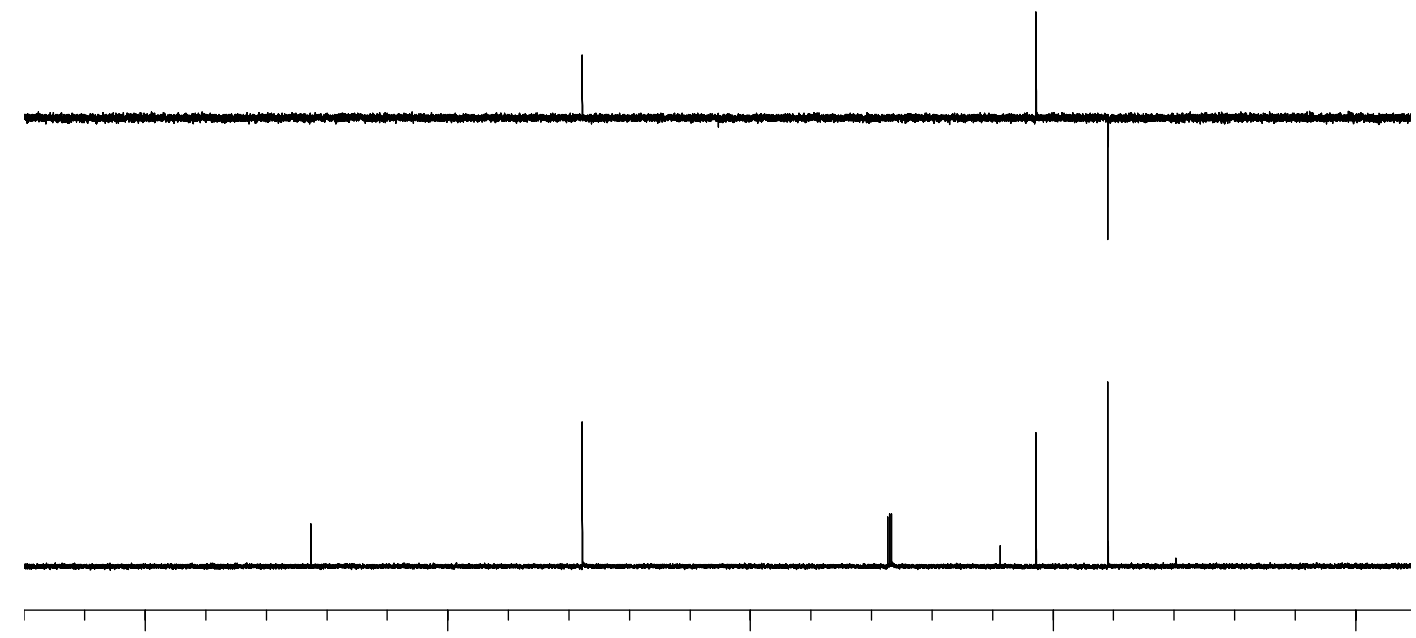

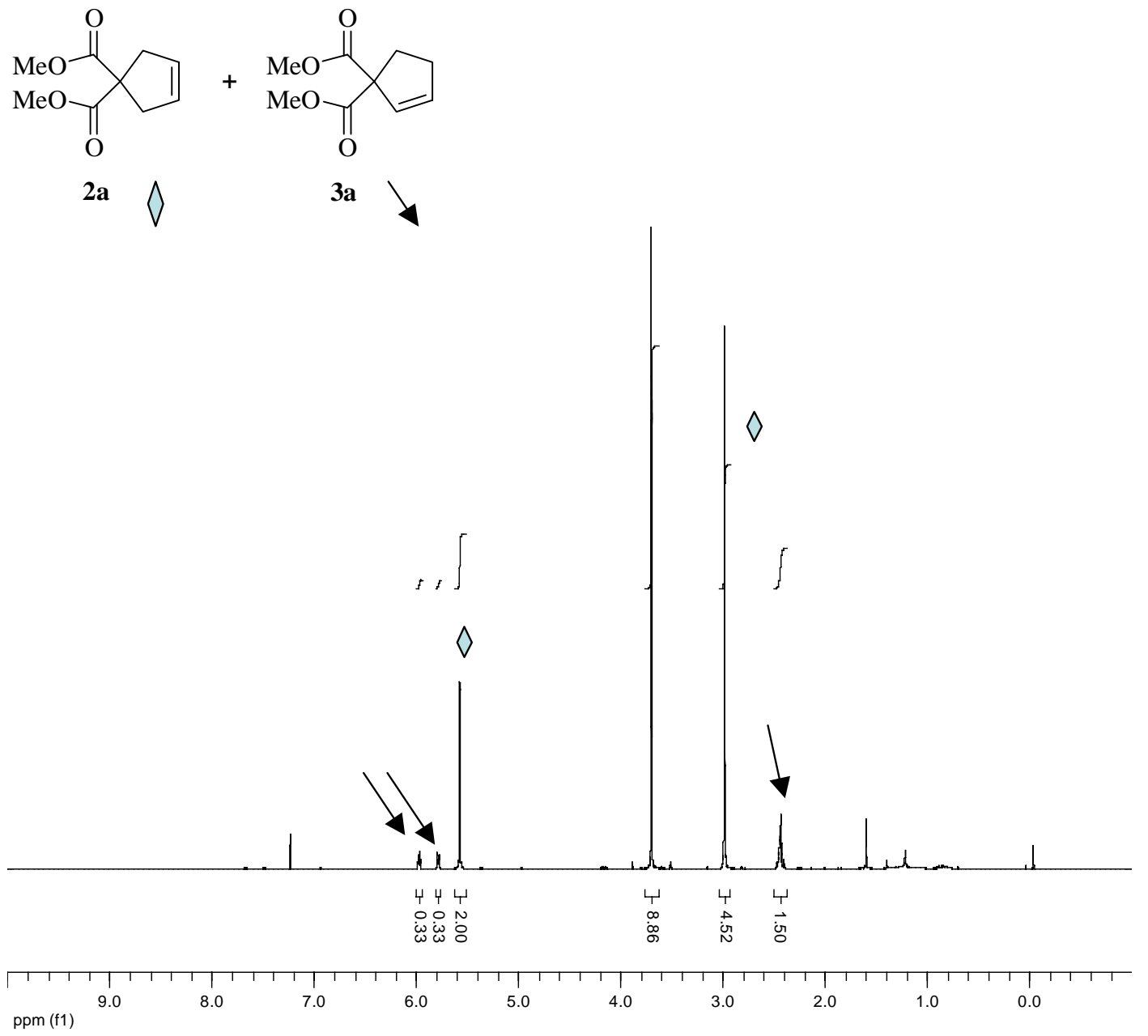

\# $\mathrm{H}_{2} \mathrm{O}$ 


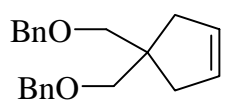

2b
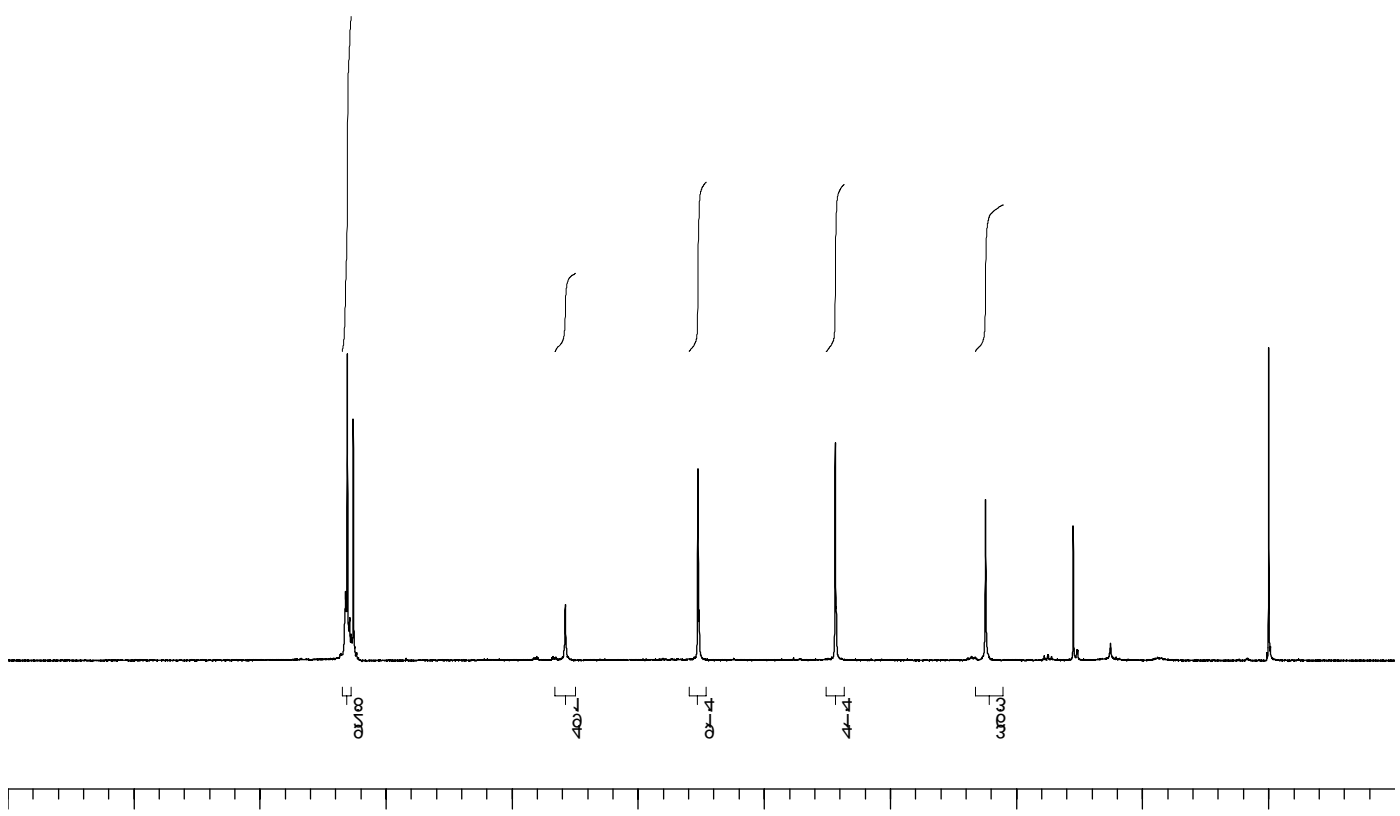

* $\mathrm{H}_{2} \mathrm{O}$
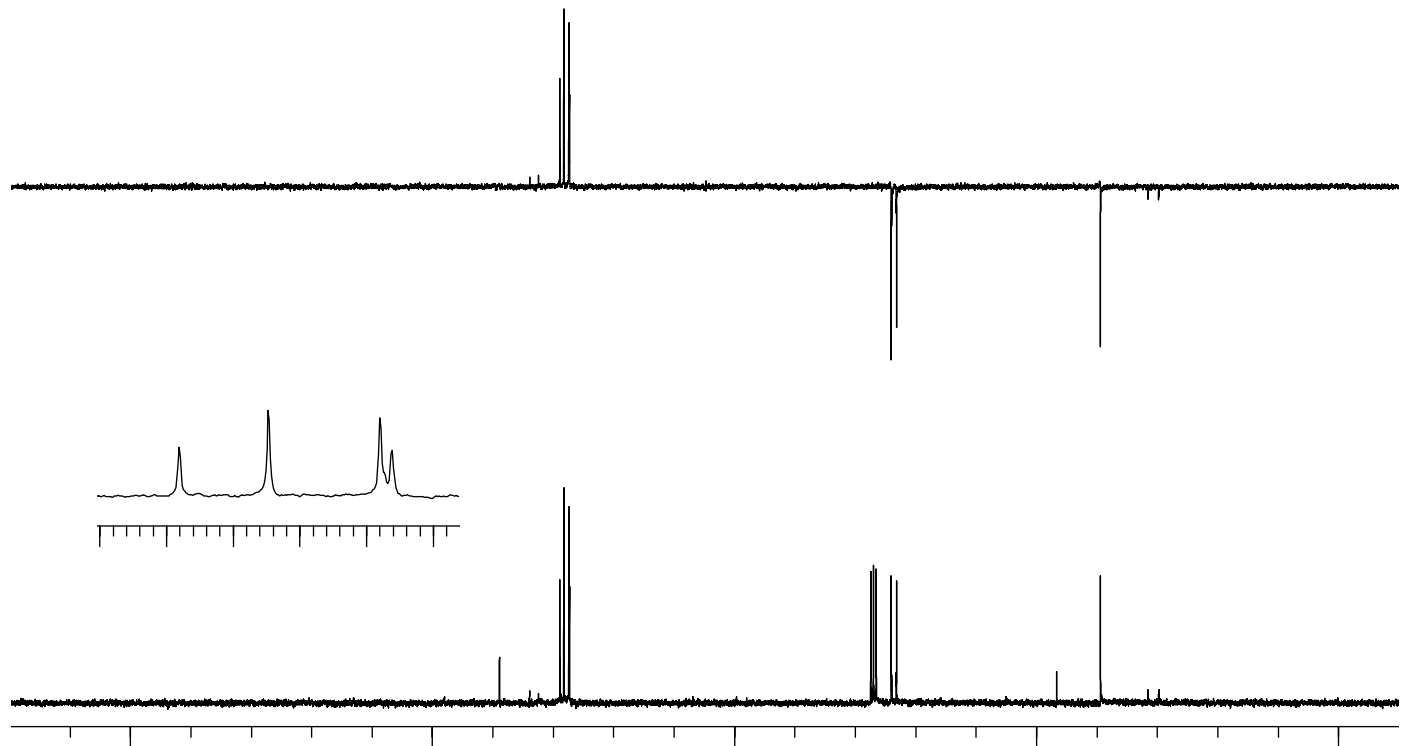


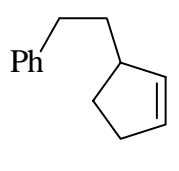

2c

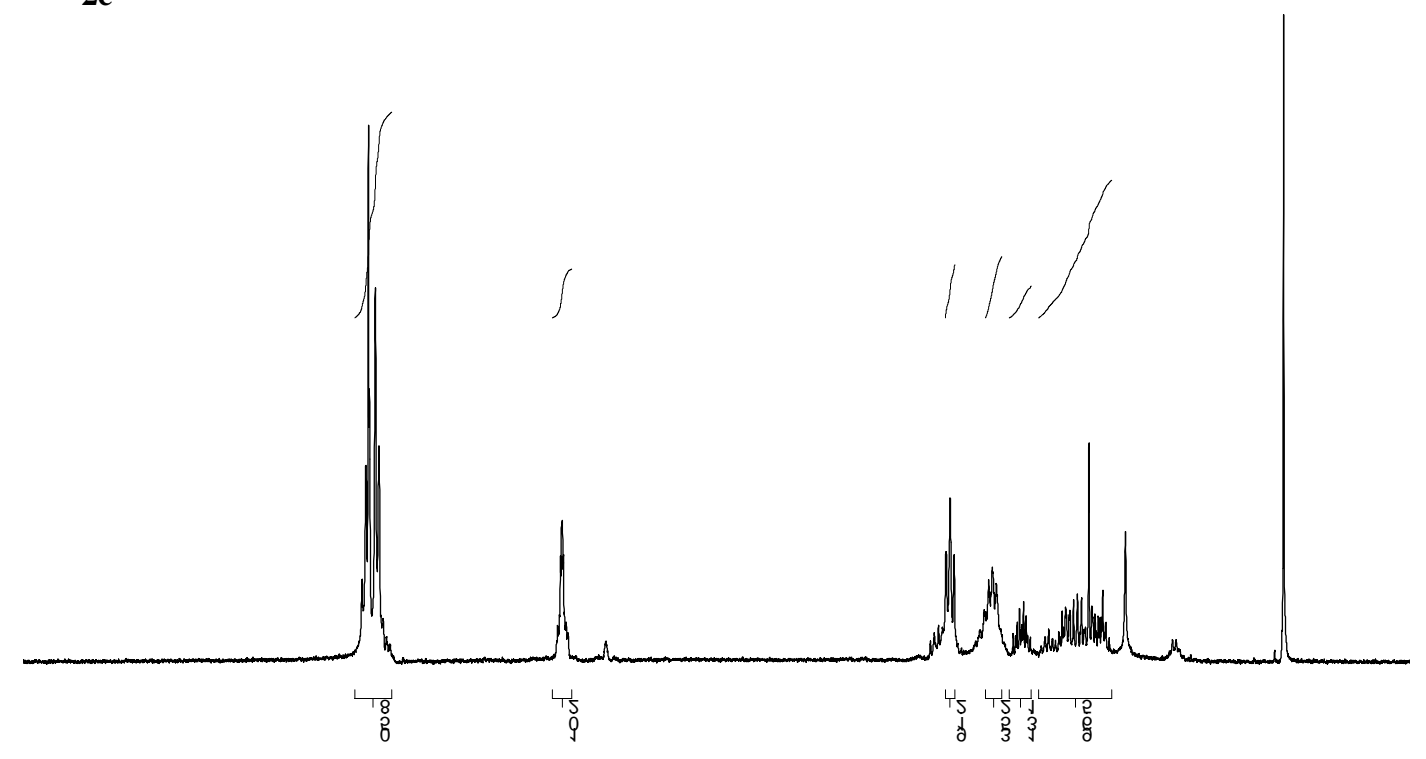

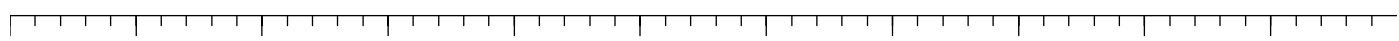

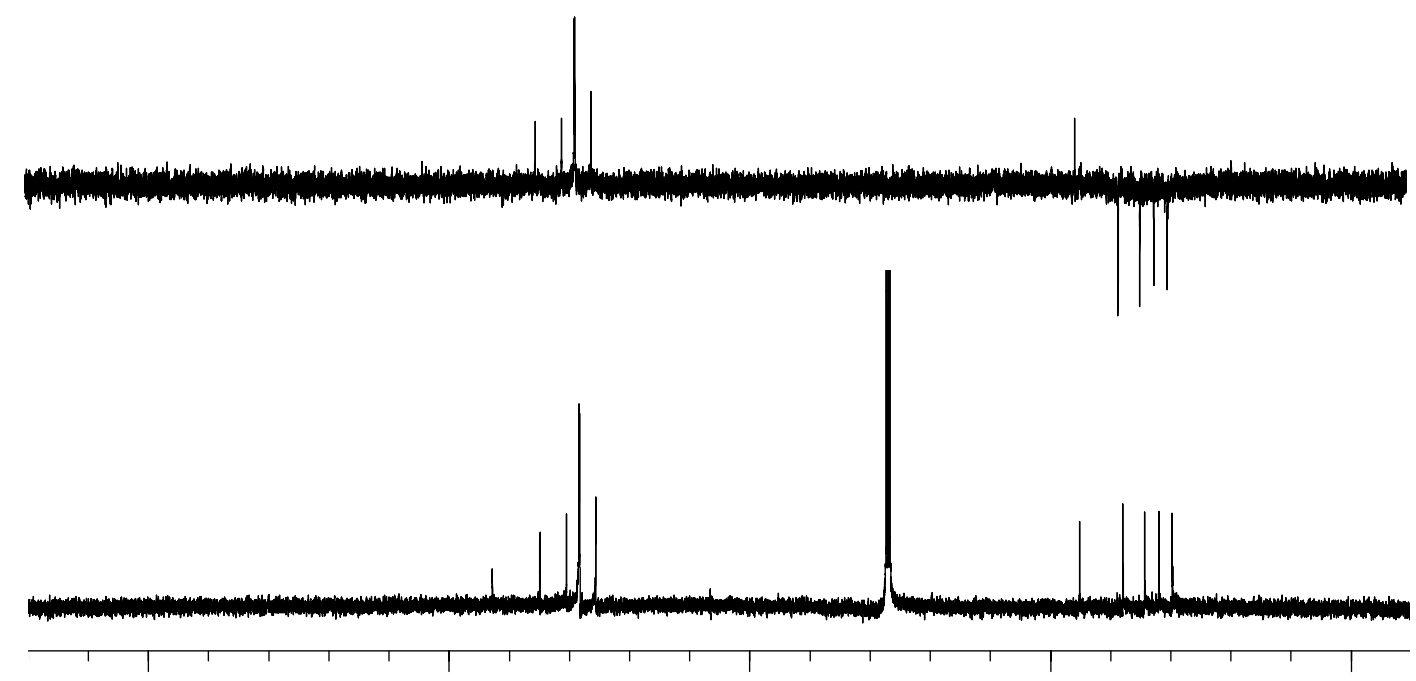


$\mathrm{Ts}-\mathrm{N}$

2d

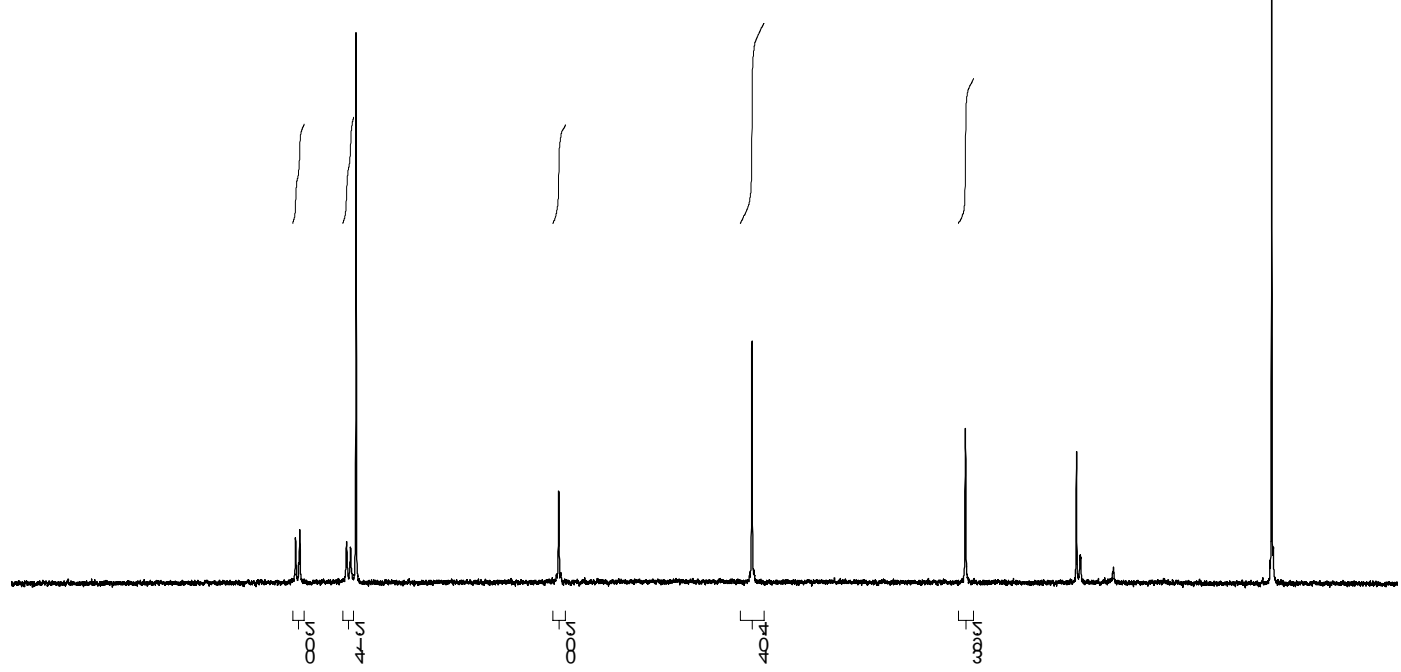

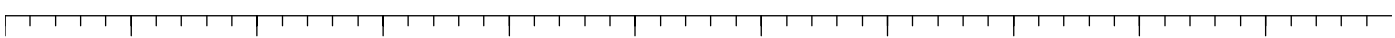

$* \mathrm{H}_{2} \mathrm{O}$

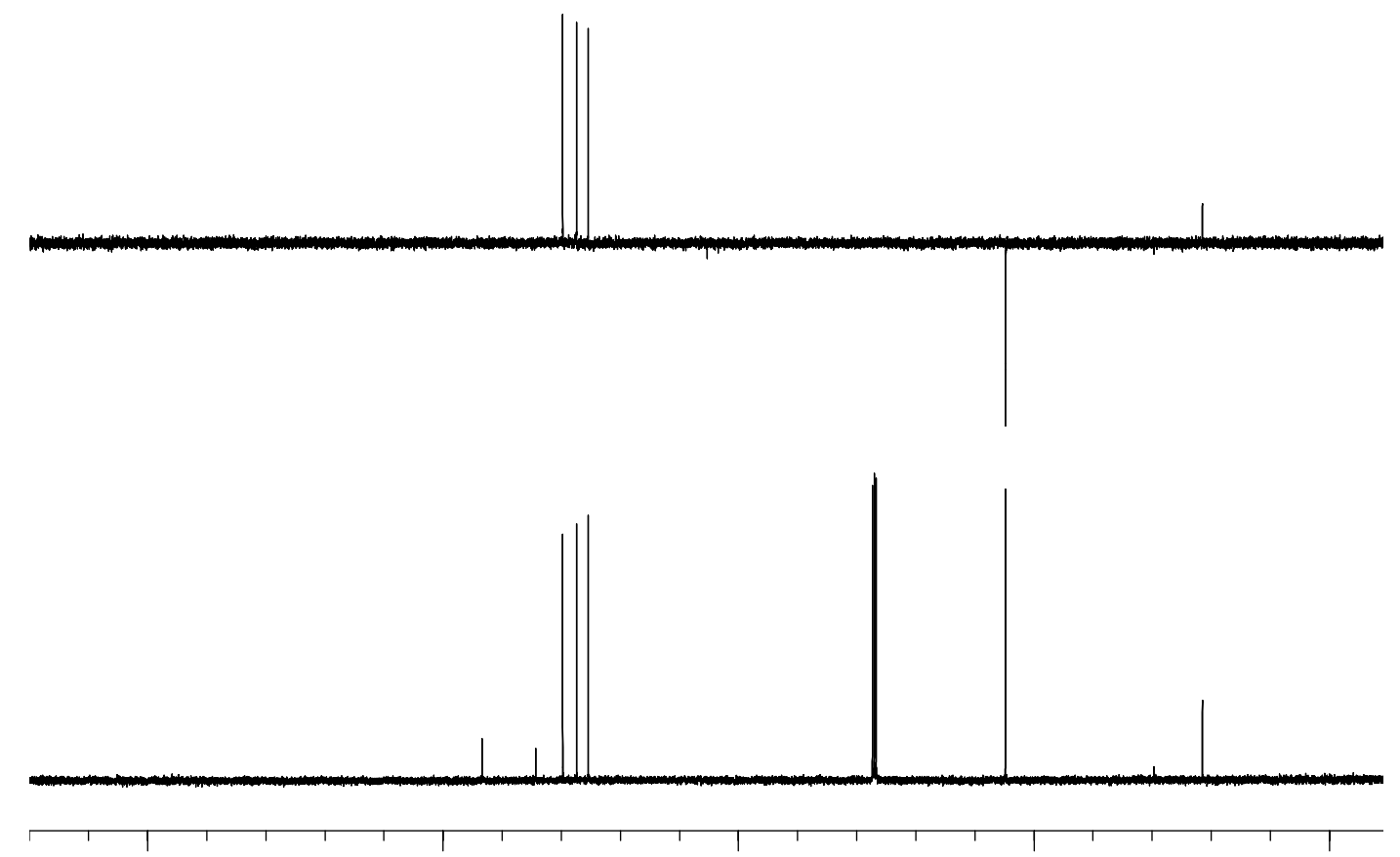




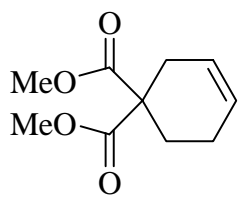

$2 \mathrm{e}$
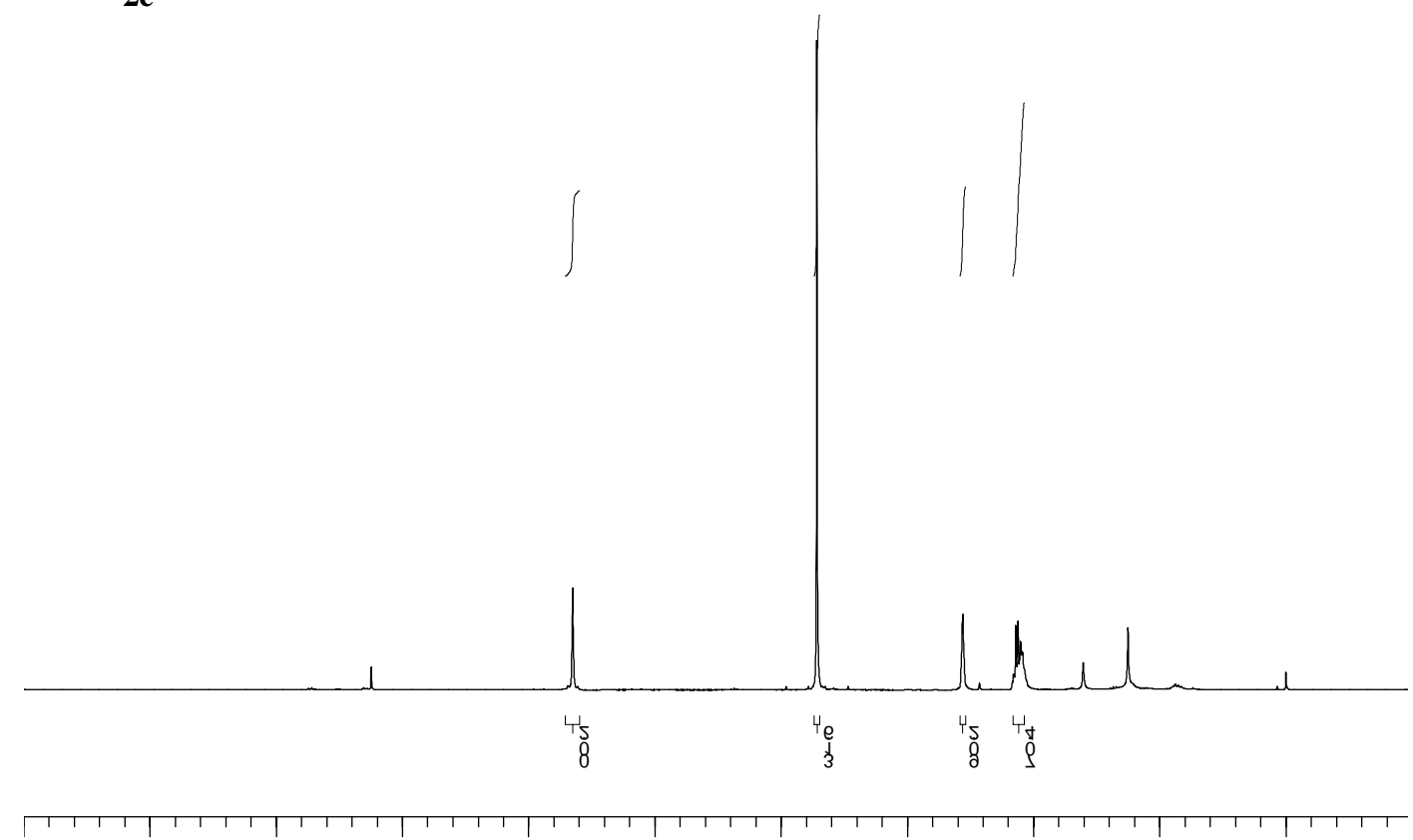

$* \mathrm{H}_{2} \mathrm{O}$

+Solvent impurity
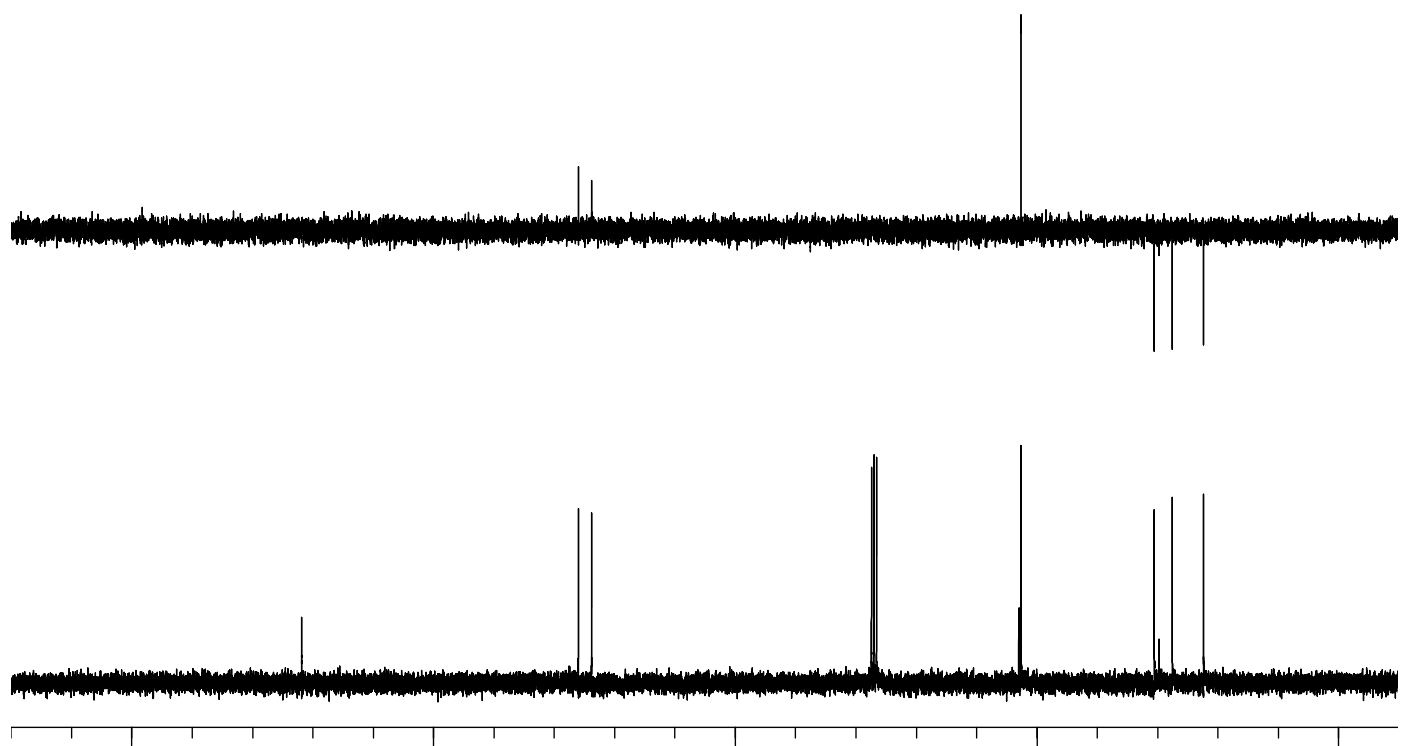
S16

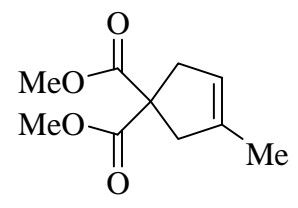

5
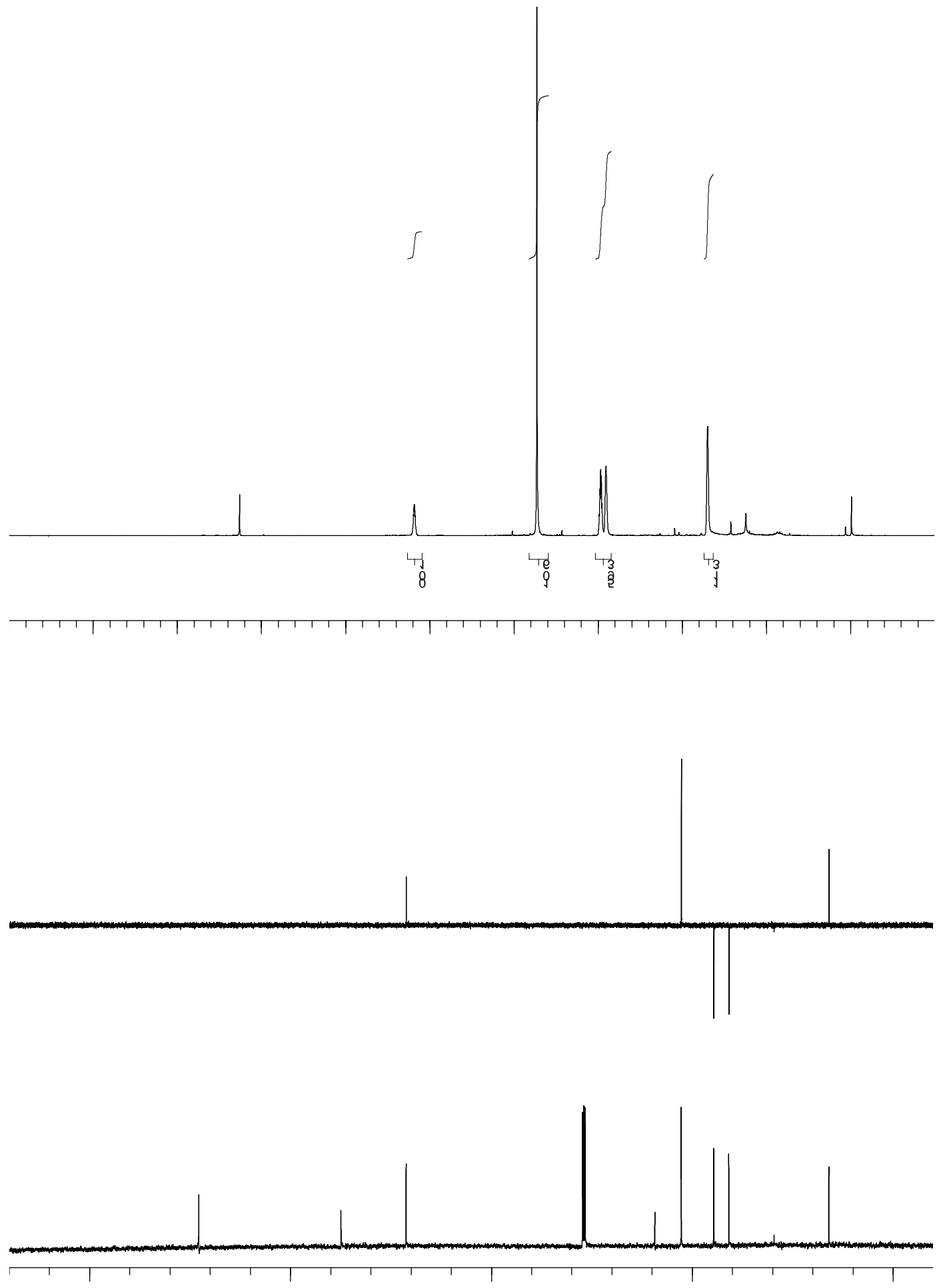


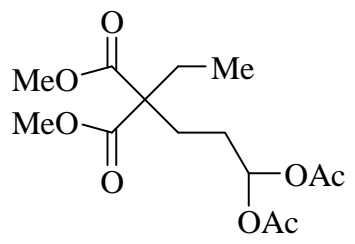

6

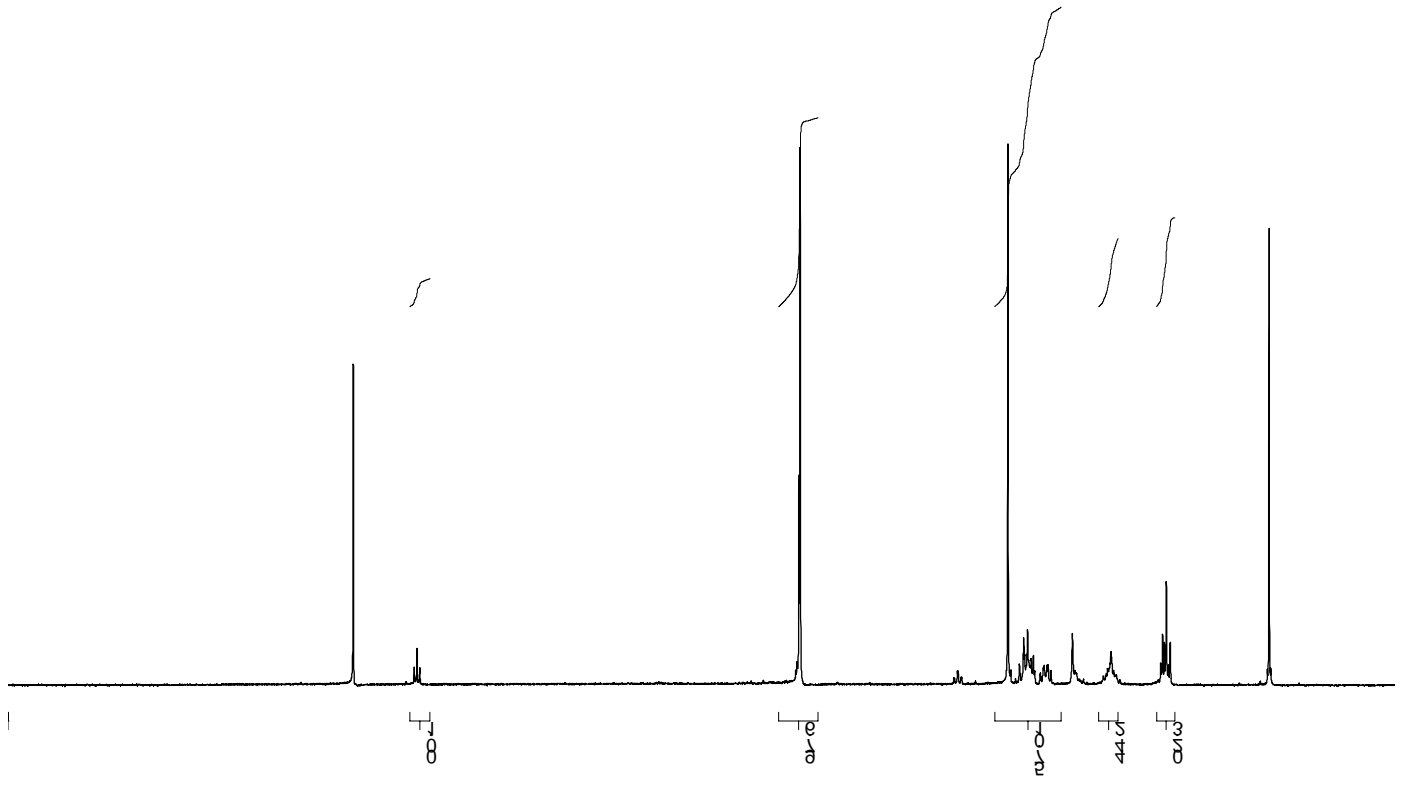

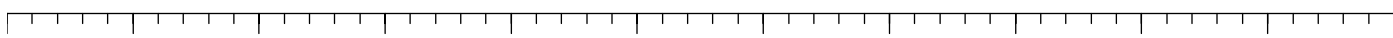

$* \mathrm{H}_{2} \mathrm{O}$

+ Impurity
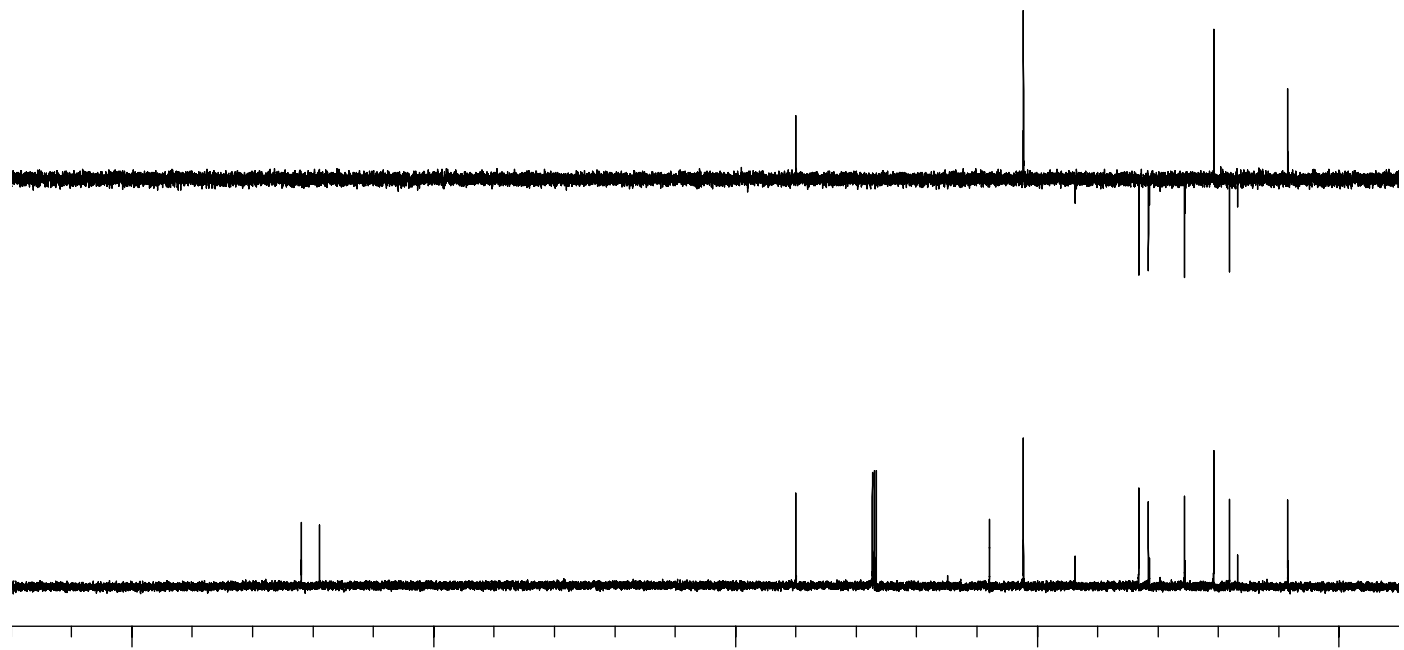


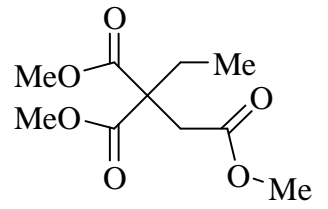

8a
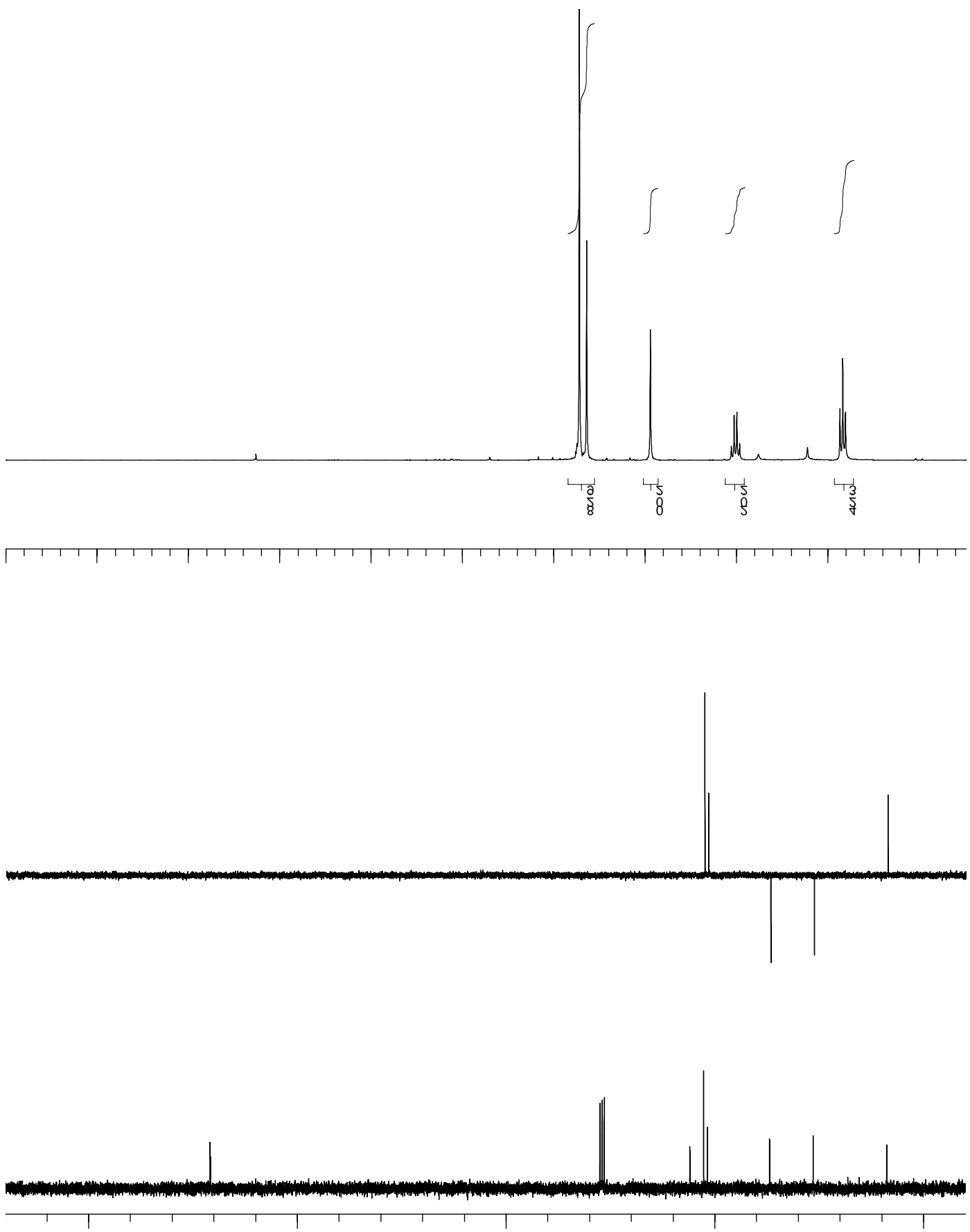


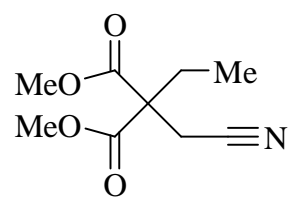

$8 b$
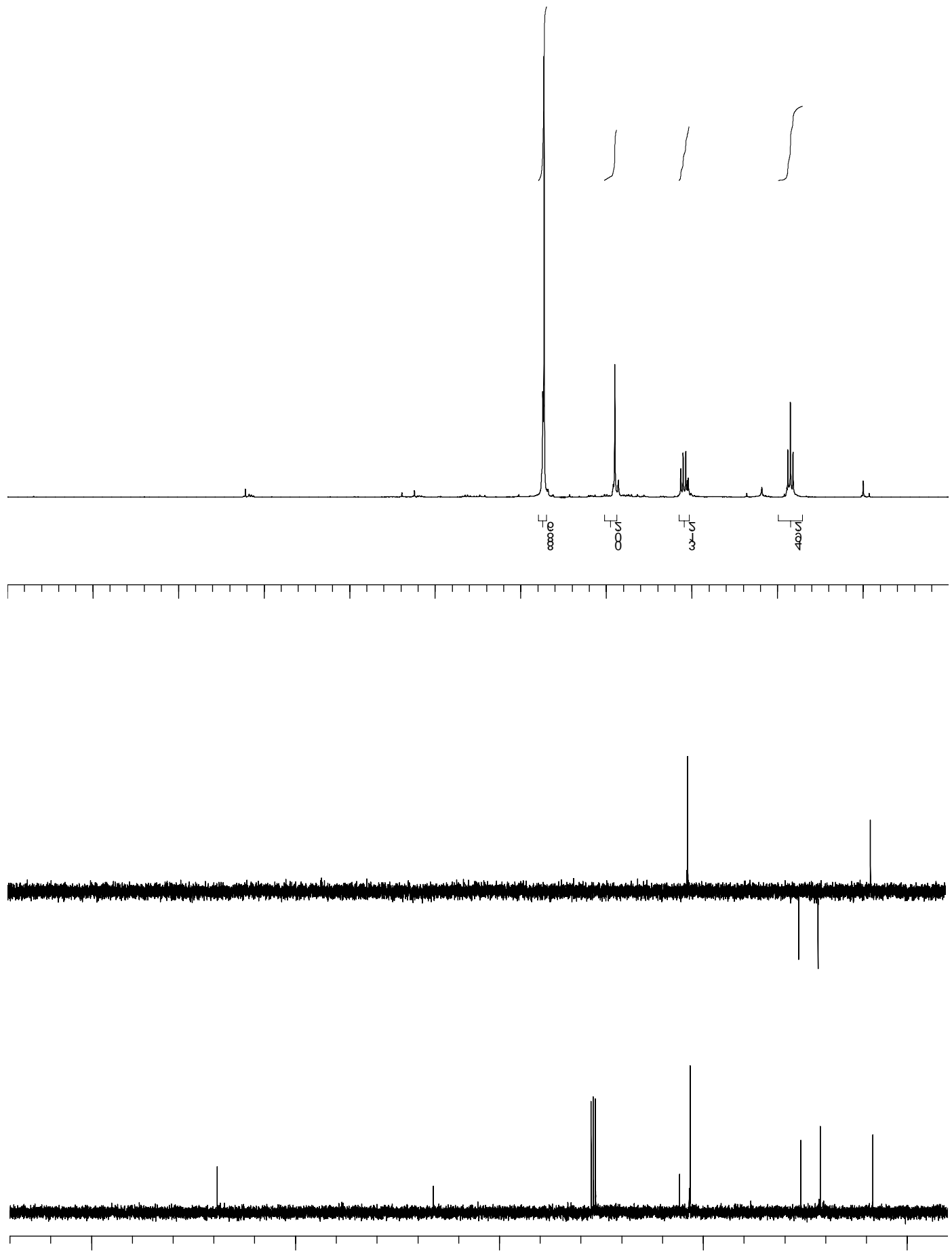


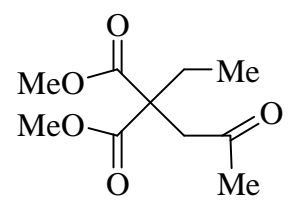

$8 c$

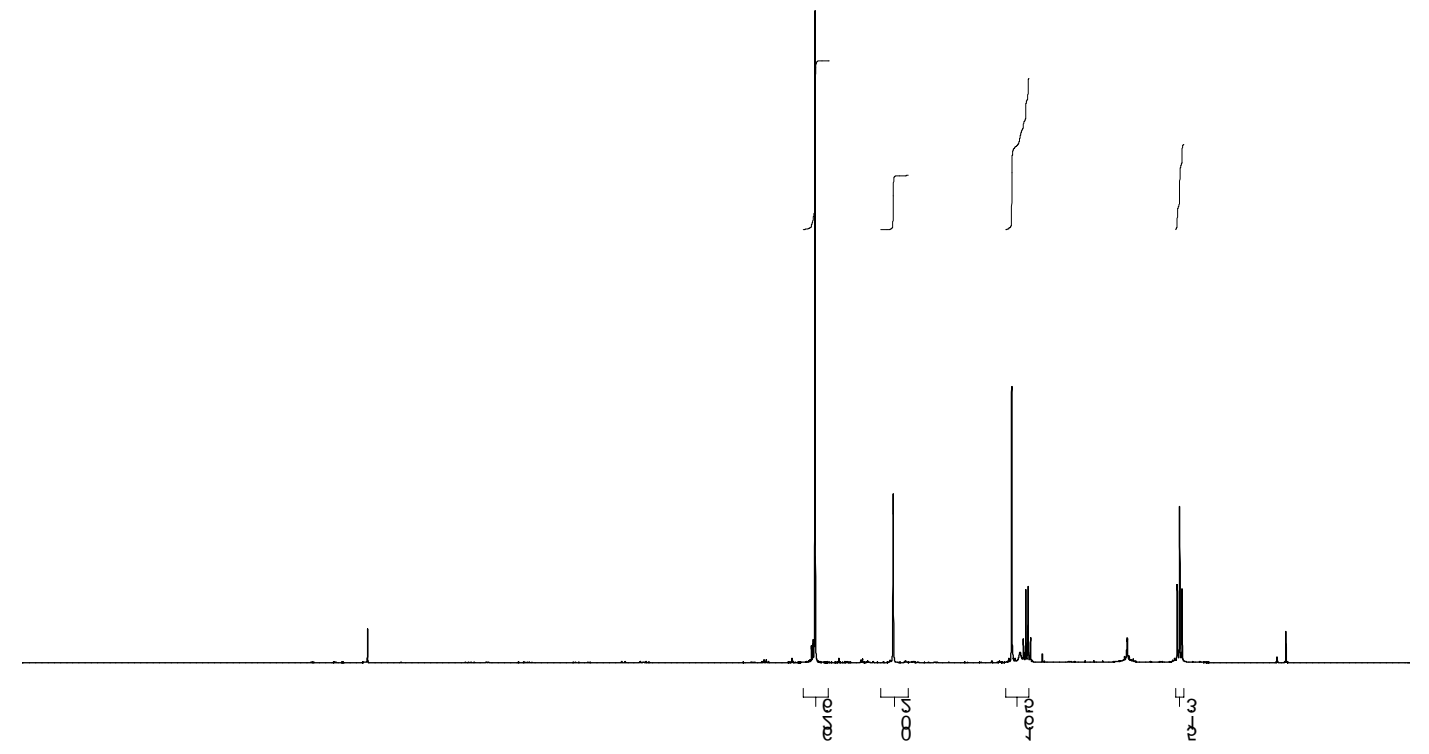

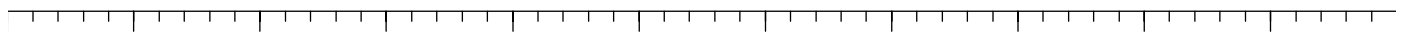
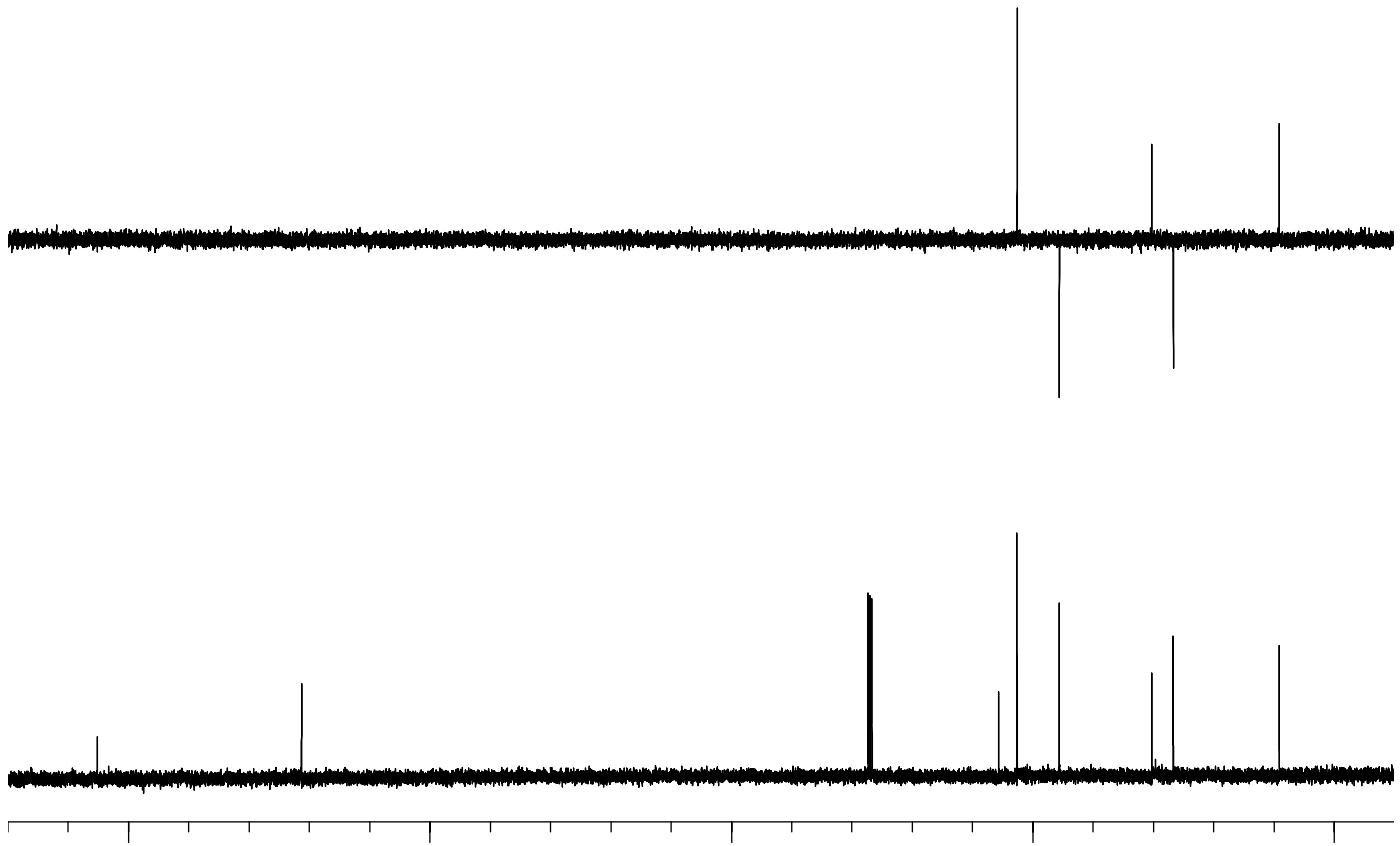


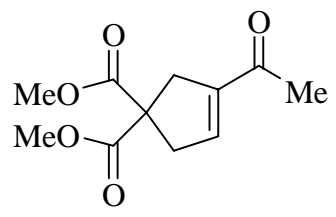

10
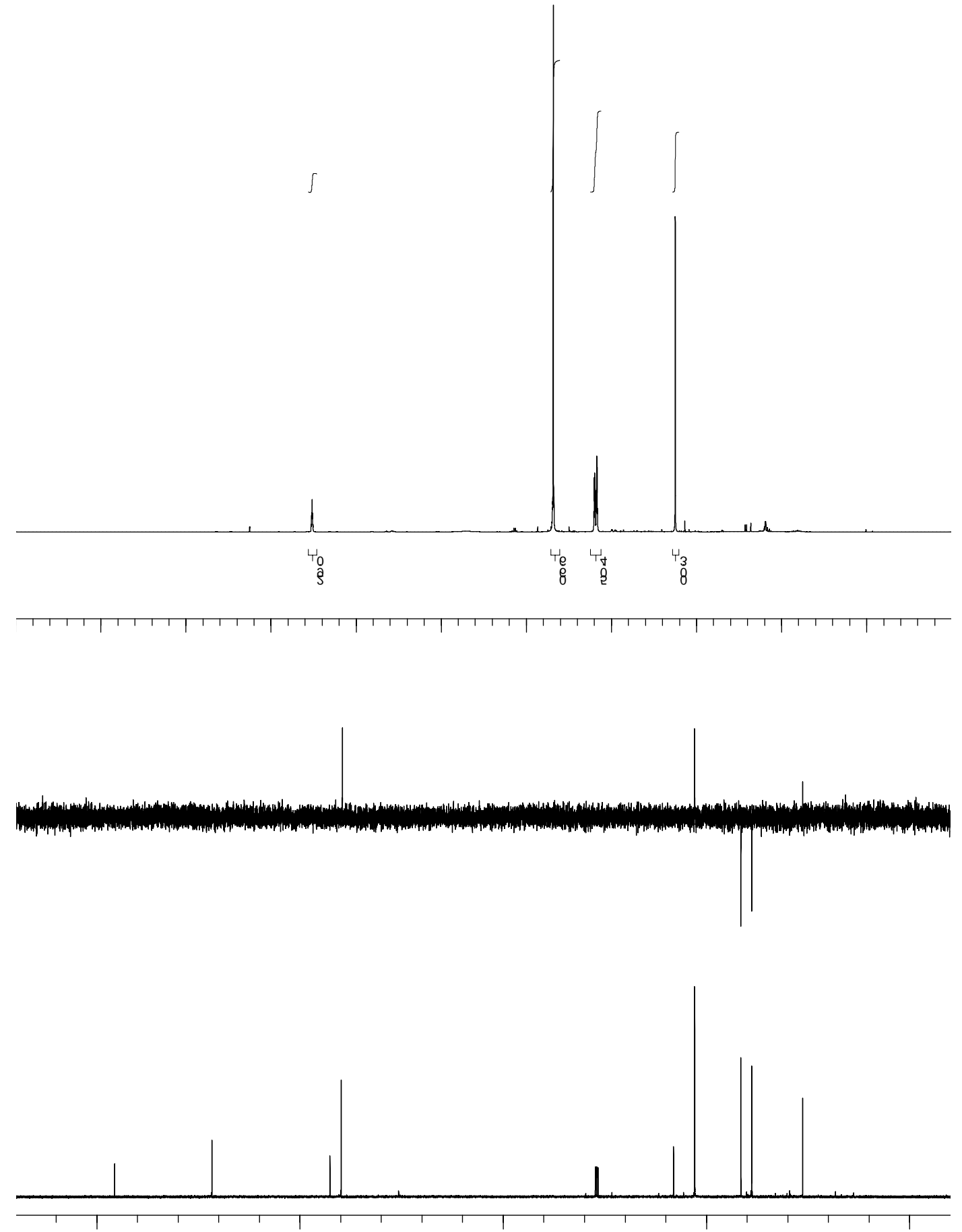


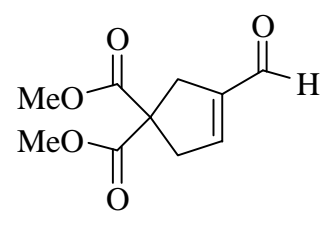

11a
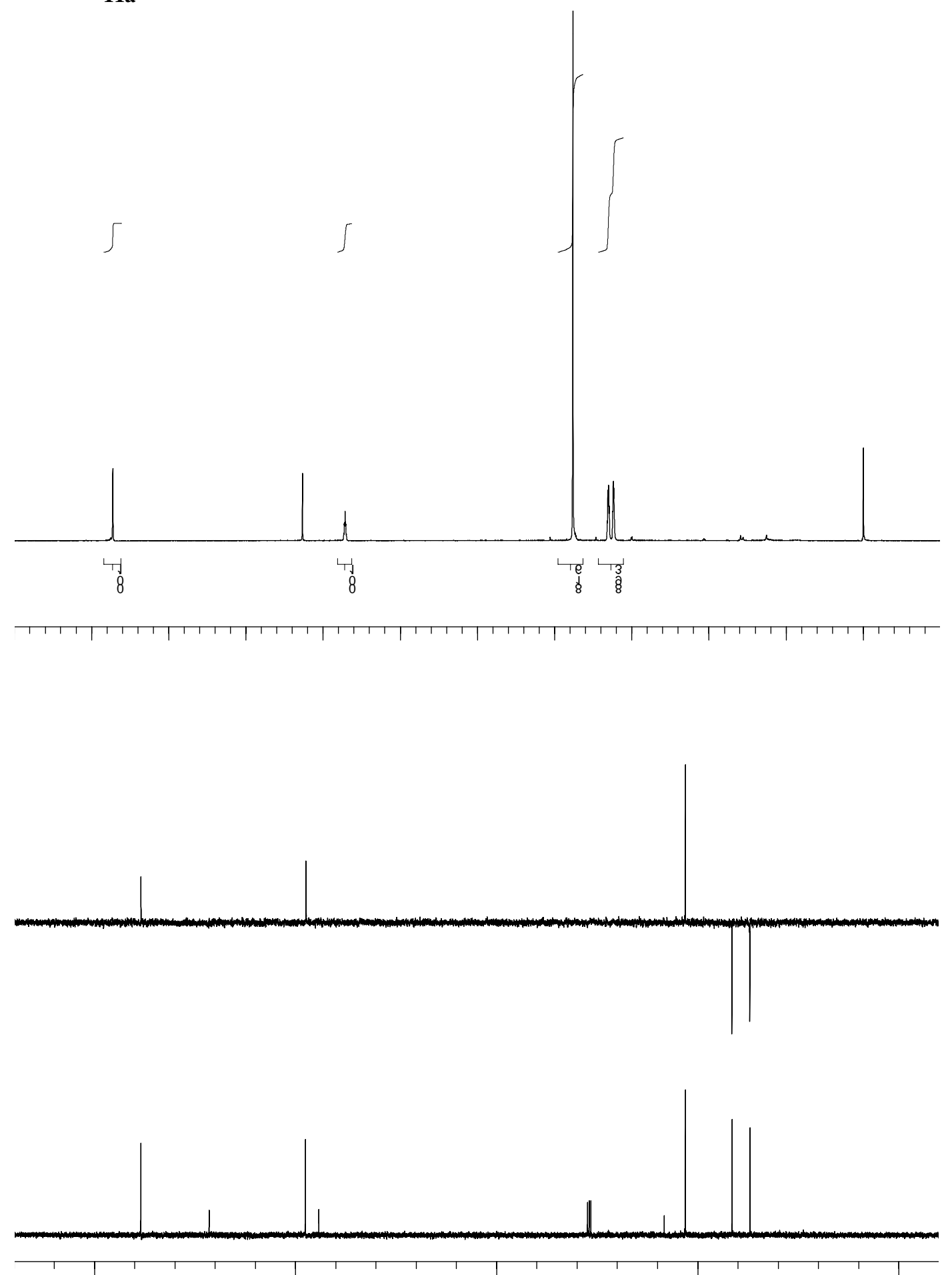


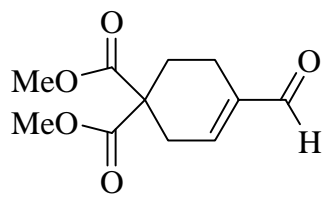

11e

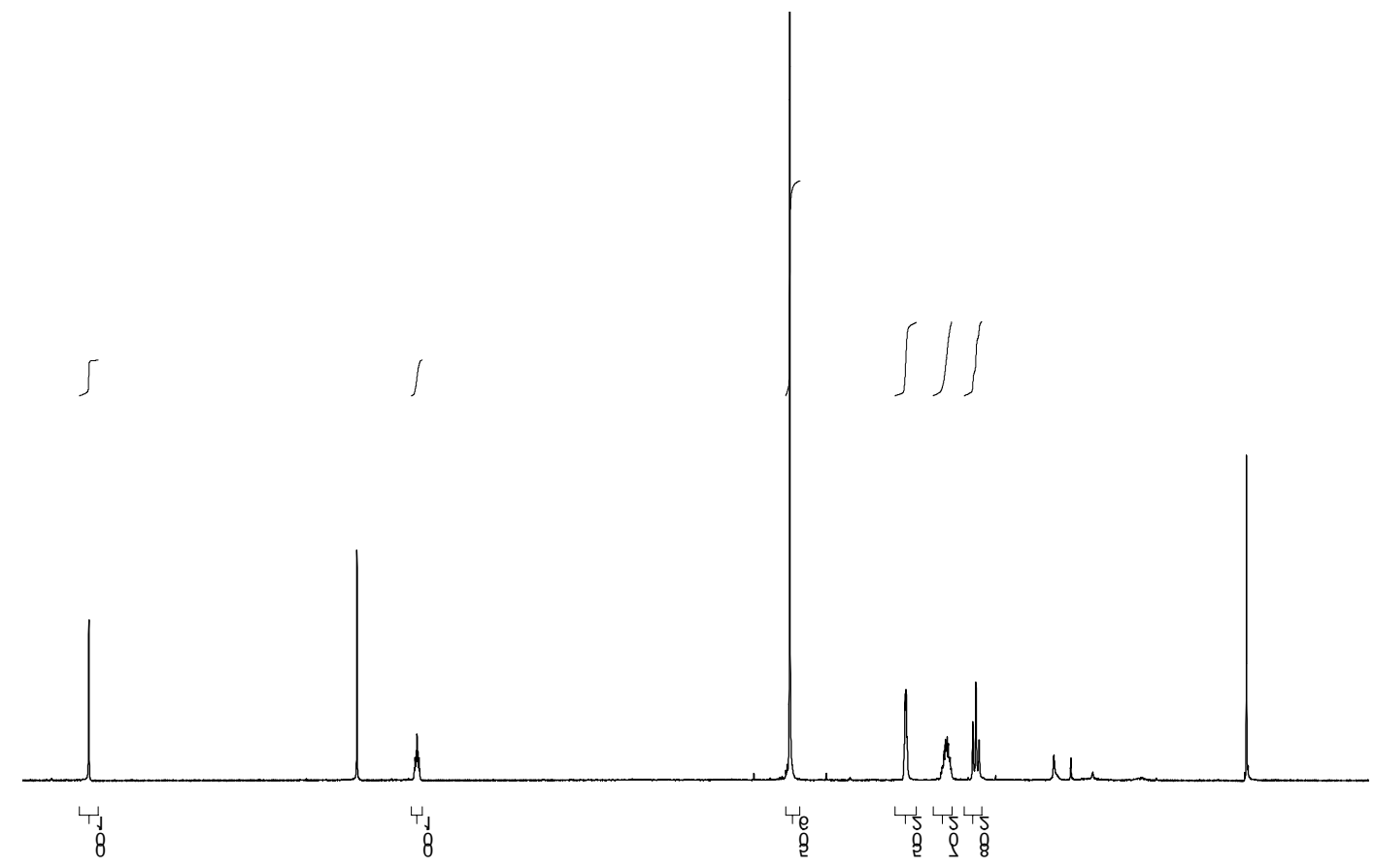

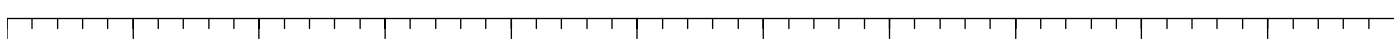
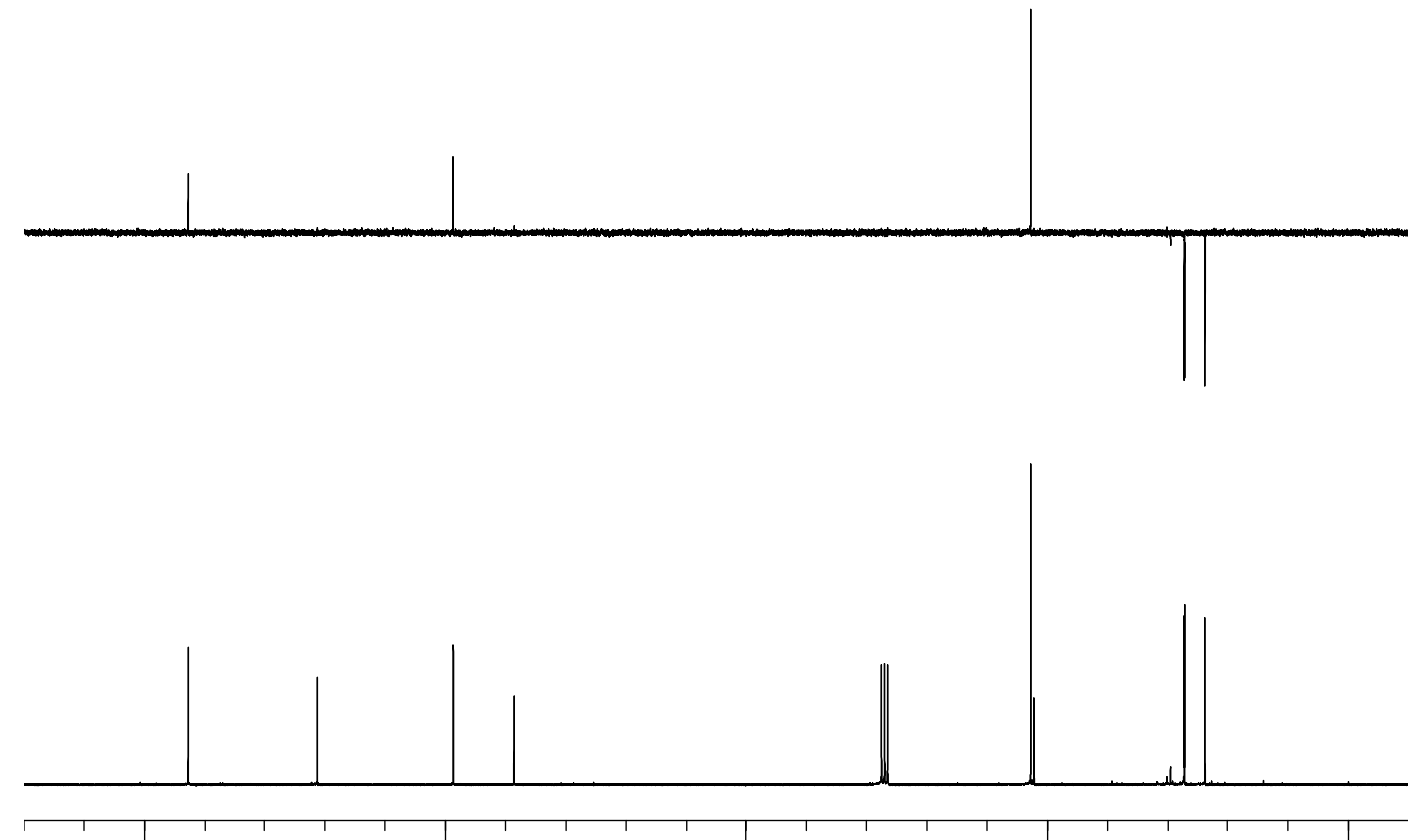


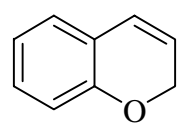

13a
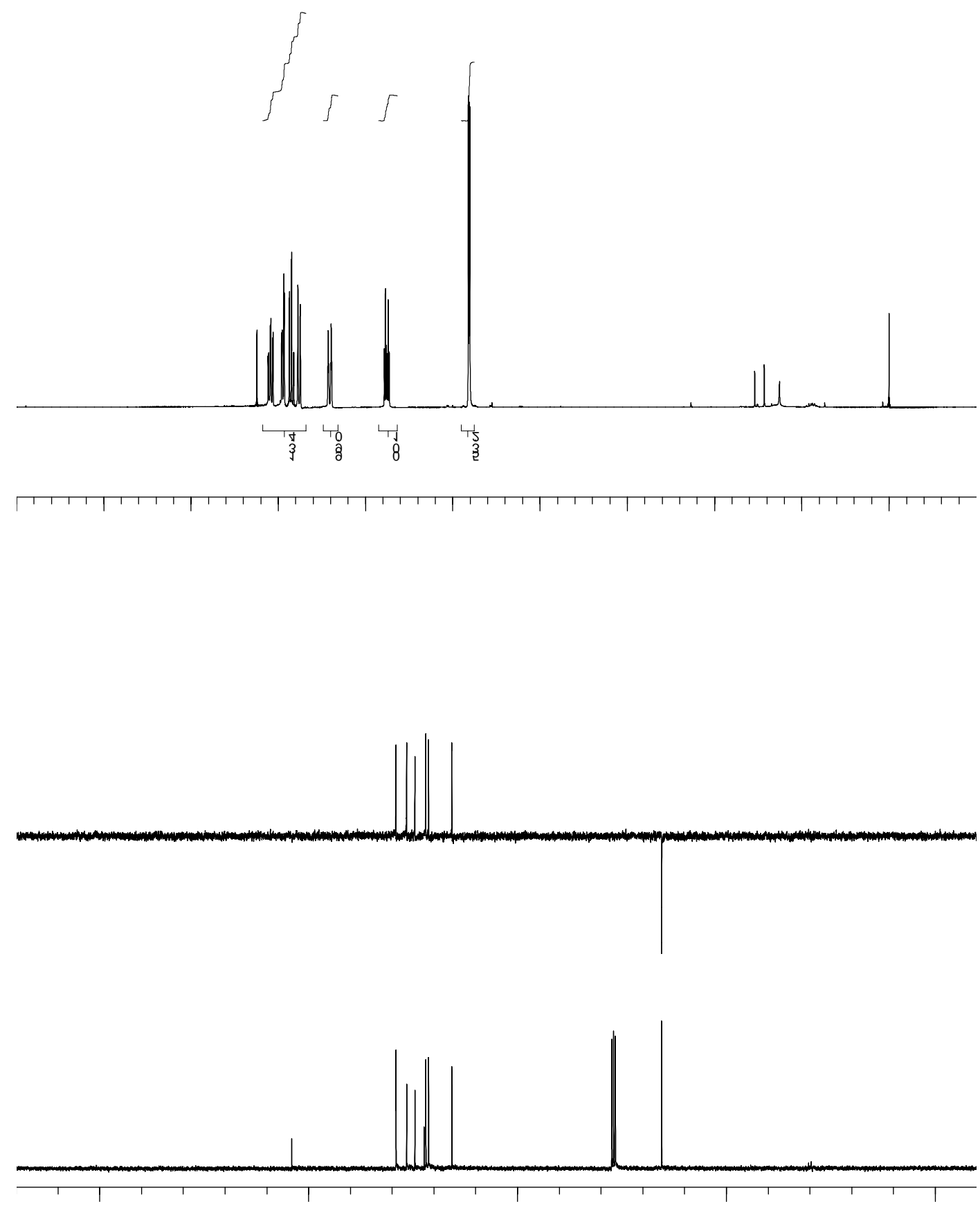


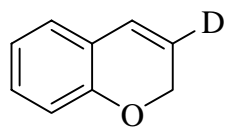

d-13
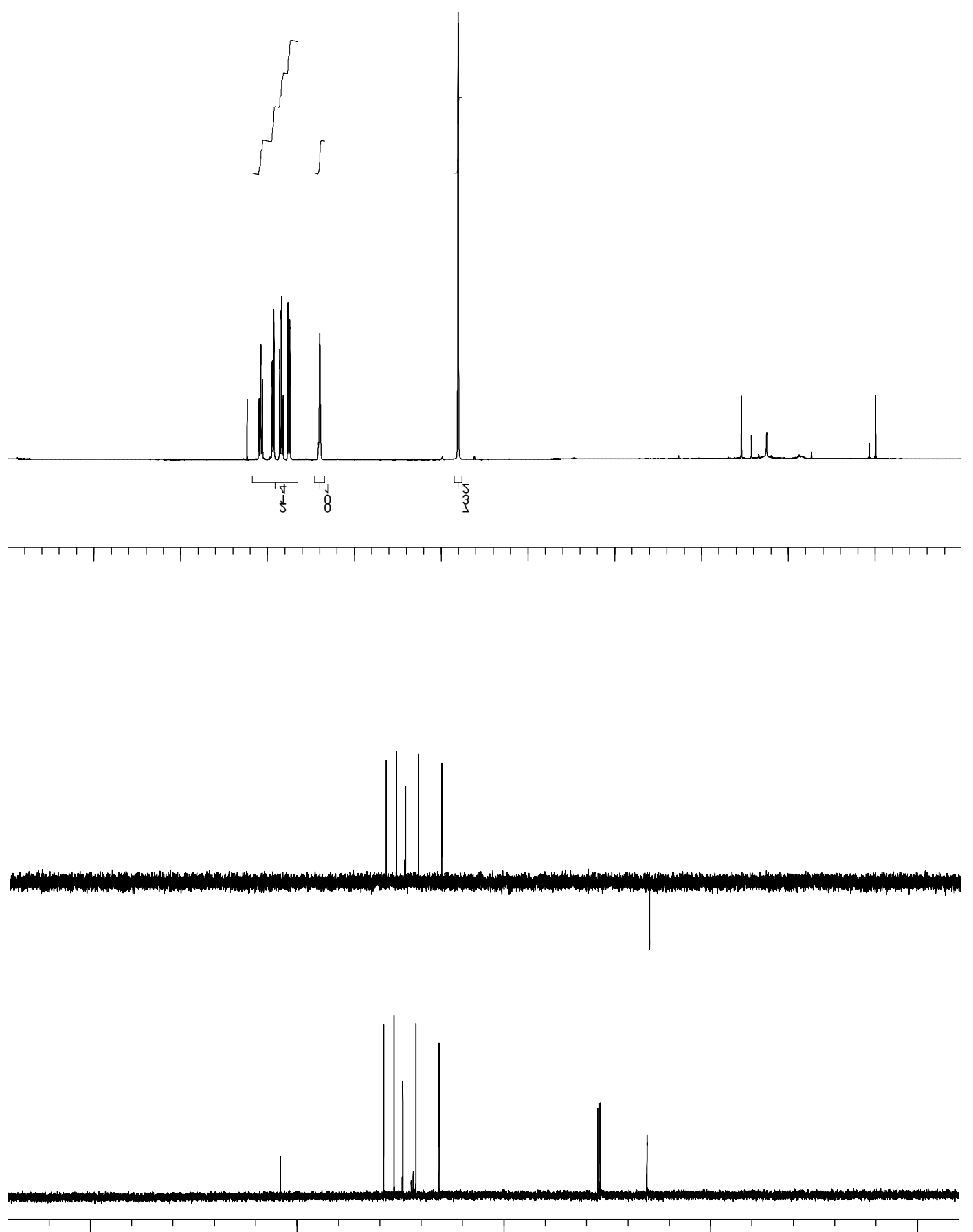

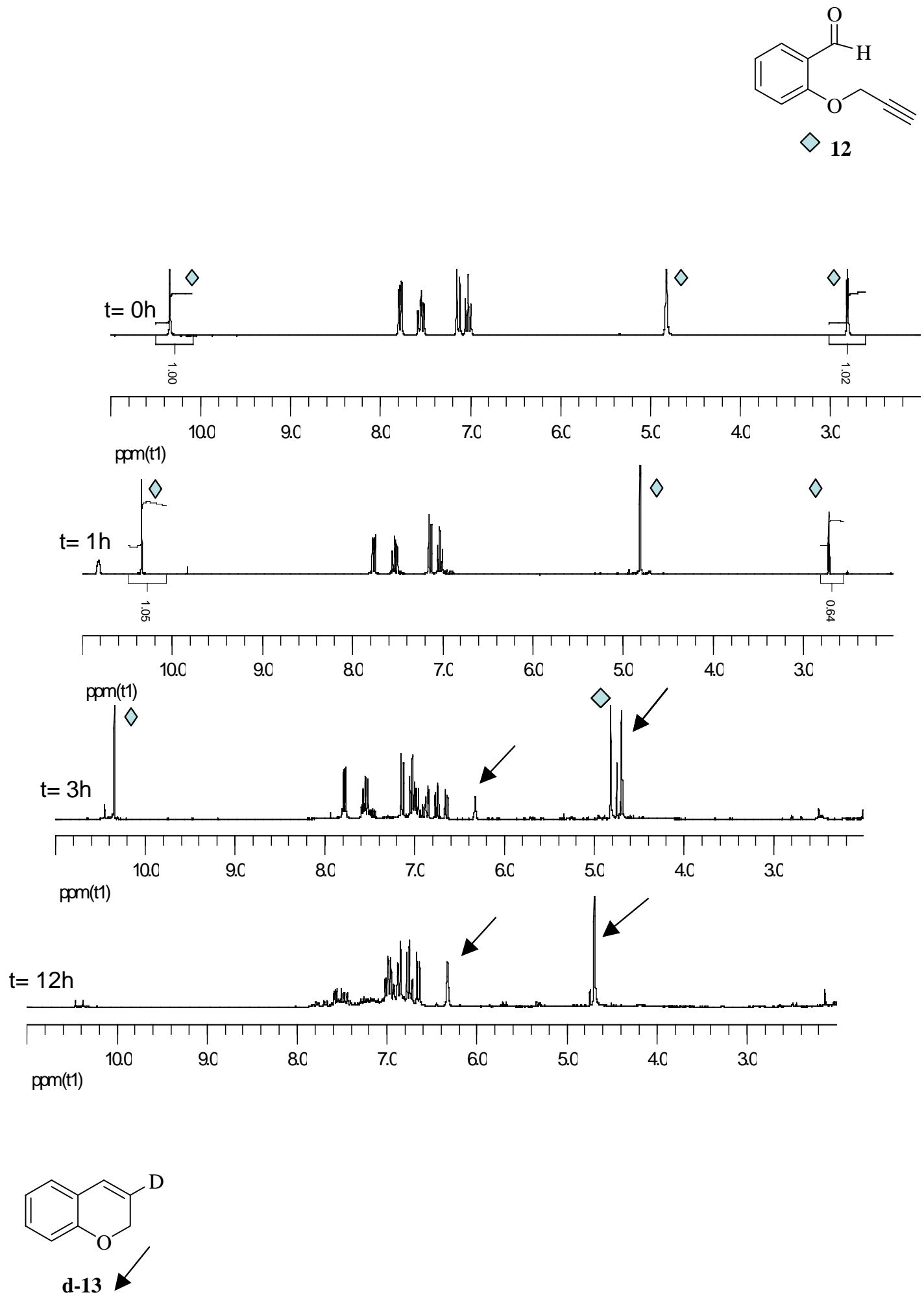


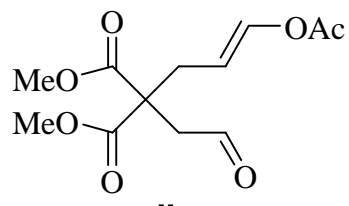

IIa

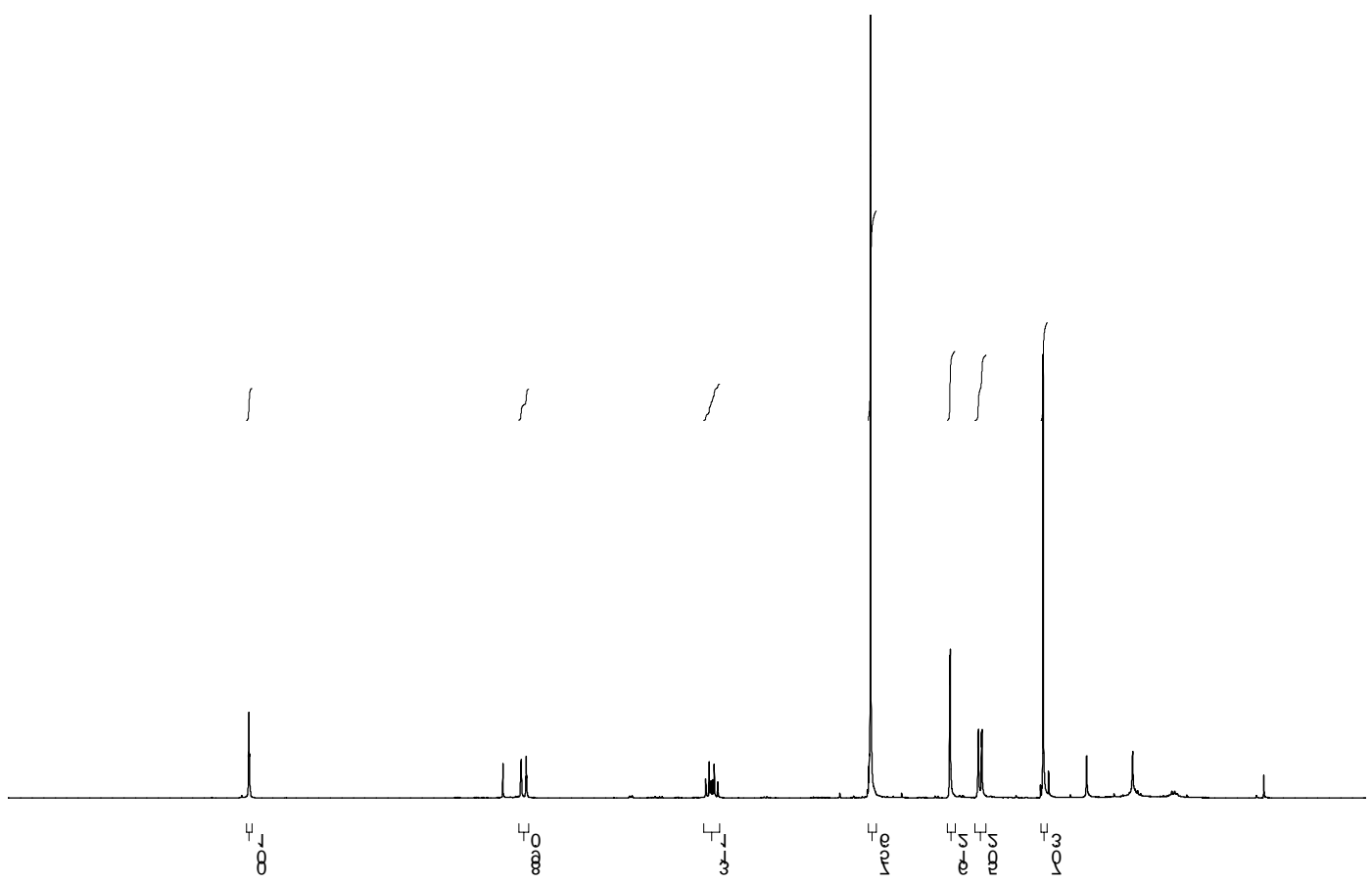

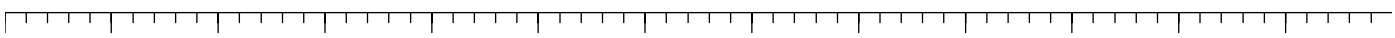
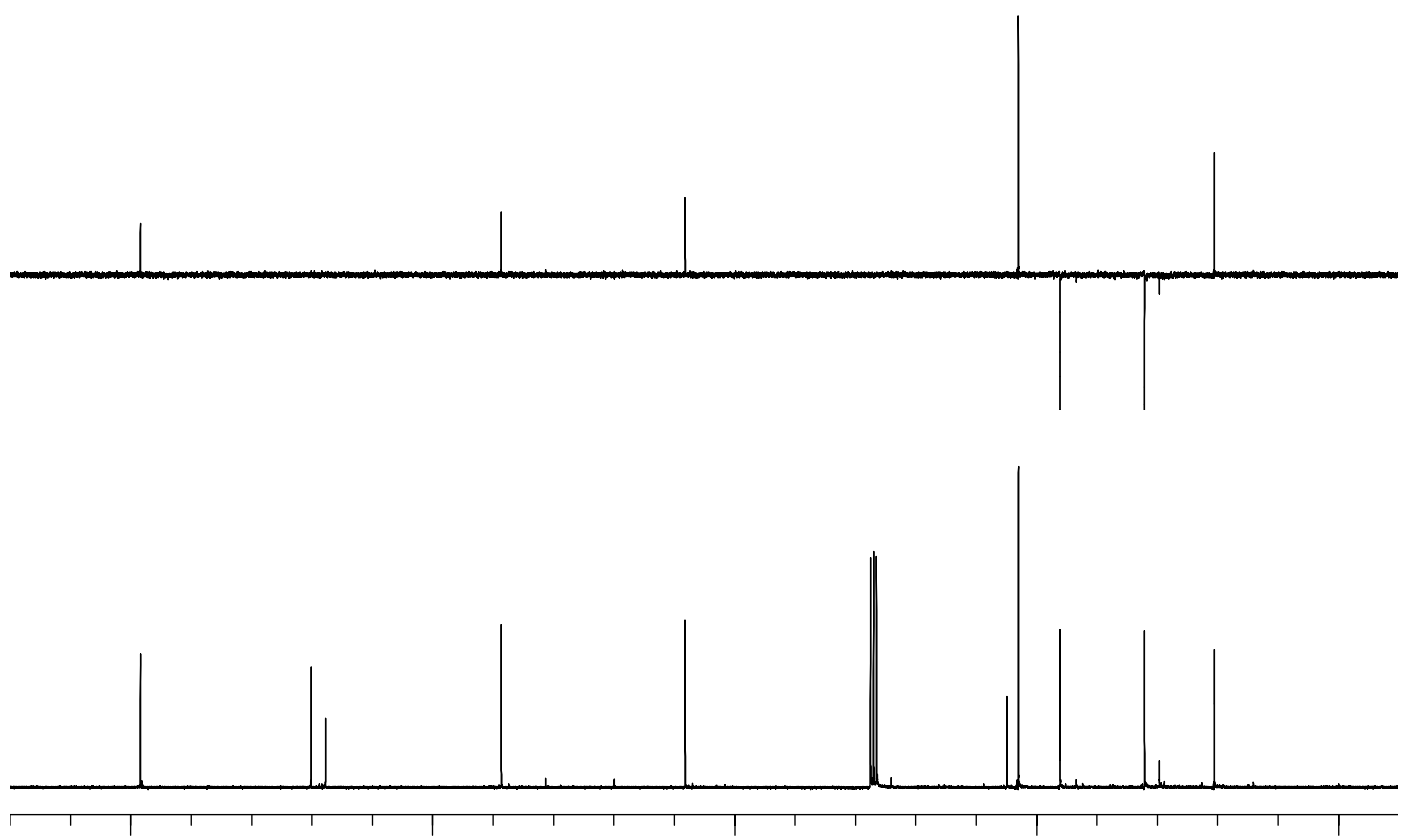